\title{
ON THE DETONATION OF A COMBUSTIBLE GAS \\ BY
}

ROBERT A. GARDNER ${ }^{1}$

\begin{abstract}
This paper is concerned with the existence of detonation waves for a combustible gas. The equations are those of a viscous, heat conducting, polytropic gas coupled with an additional equation which governs the evolution of the mass fraction of the unburned gas (see (1)). The reaction is assumed to be of the simplest form: $A \rightarrow B$, i.e., there is a single product and a single reactant. The main result (see Theorem 2.1) is a rigorous existence theorem for strong, and under certain conditions, weak detonation waves for explicit ranges of the viscosity, heat conduction, and species diffusion coefficients. In other words, a class of admissible "viscosity matrices" is determined.

The problem reduces to finding an orbit of an associated system of four ordinary differential equations which connects two distinct critical points. The proof employs topological methods, including Conley's index of isolated invariant sets.
\end{abstract}

1. Introduction. The equations which describe the evolution of a combustible gas (see (1)) display a rich variety of nonlinear phenomena, including those encountered in reaction-diffusion equations and in shock wave theory. In particular, there are two distinct mechanisms that can lead to the formation of combustion waves. Slow combustion, or deflagration, occurs when an exothermic chemical reaction is initiated in a heat conducting gas. The subsequent diffusion of liberated heat into the surrounding medium leads to the formation of a flame which propagates into the unburned region. The fluid dynamics of the gas mixture plays a negligible role in this regime. Fast combustion, or detonation, occurs in a dramatically different manner. Here, the process is initiated by a strong fluid dynamical shock layer which propagates into the unburned region. If the shock is sufficiently strong, the gas will be heated up above its ignition temperature, causing the gas to burn in a reaction zone behind the shock. The released heat of reaction can have a significant effect on the fluid flow. In particular, the liberated heat sustains the propagation of the wave front in much the same way that a piston causing a compression wave can sustain a purely fluid dynamical shock. It is reasonable to expect that the dissipation of energy due to viscosity, heat conduction and species diffusion, counteracts the sharp release of chemical energy and can therefore inhibit the detonation process. The problem to be investigated here is to determine explicit conditions on these dissipative mechanisms under which detonation profiles will exist.

Received by the editors June 1, 1982. Presented at the A.M.S. Summer Research Conference on Nonlinear Partial Differential Equations, Durham, New Hampshire, 1982.

1980 Mathematics Subject Classification. Primary 35K55, 58E05, 34L35.

${ }^{1}$ Research partially supported by N.S.F. contract MCS-8101644.

(C)1983 American Mathematical Society 0002-9947/82/0000-0642/\$22.50 
The hypotheses (cf. §2) under which the main theorem is proved are that the gas mixture is polytropic and that its molecular weight and specific heat are (approximately) independent of its chemical composition. It is also assumed that for a given state $\left(\tau_{R}, T_{R}\right)$ of the unburned gas (where $\tau$ is specific volume and $T$ is temperature), that the wave velocity, $\sigma$, is large enough so that a fluid dynamaical shock profile heats the gas up above its ignition temperature, $T_{i}>T_{R}$. The state $\left(\tau_{R}, T_{R}\right), \sigma$, and a third parameter $q_{0}$, determine two possible states $\left(\tau_{L^{*}}, T_{L^{*}}\right)$ and $\left(\tau_{L}^{*}, T_{L}^{*}\right)$ for the burned gas; $q_{0}$ is the liberated energy/unit mass of the chemical reaction. It will also be assumed that the temperature along a shock profile in the burned gas always exceeds $T_{i}$, i.e., $T_{i}<T_{L^{*}}<T_{L}^{*}$. (We restrict attention to this case since it presents the most interesting mathematical questions. Other cases which can occur are when $T_{L^{*}}<T_{i}<T_{L}^{*}$, and when $T_{L^{*}}<T_{L}^{*}<T_{i}$; see $\S 6$.E for further remarks about these cases.) These hypotheses determine a region of admissible $\left(\sigma, q_{0}\right)$ corresponding to the shaded region in Figure 0 . (The left boundary, $q_{0}=\bar{q}_{0}(\sigma)$ corresponds to Chapman-Jouget detonation. For further remarks about this important limiting case, see §6.D.) Finally, certain restrictions must be placed on the size of the coefficients of viscosity, heat conduction, and species diffusion (cf. Theorem 2.1).

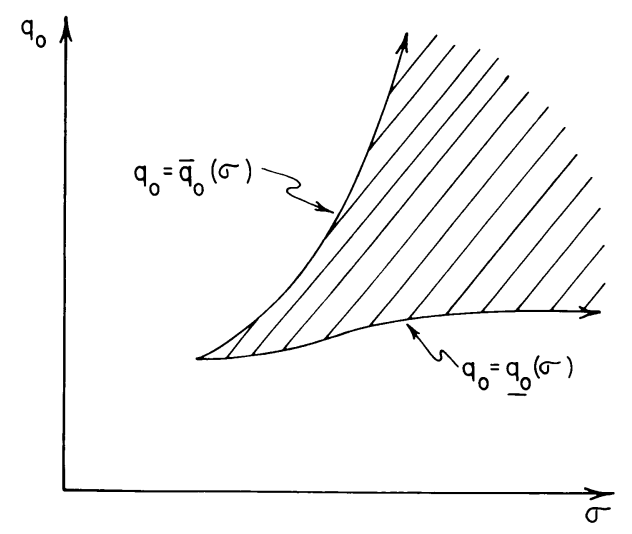

FIGURE 0

One difficulty we shall encounter is that the gas reacts at a small, but negligible rate in the unburned region; that is, the reaction rate $\phi=K \exp (-A / T)$ (where $K$ and $A$ are positive constants) is positive, unless $T=0$. The "cold boundary difficulty" is avoided by replacing the above Arrhenious rate law with ignition temperature kinetics, wherein $\phi$ is now taken to be a nondecreasing function similar to the exponential, but which vanishes identically below some ignition temperature, $T_{i}$. This appears to be an acceptable approximation (see Williams [9]), in that detonation waves obtained with ignition temperature kinetics exhibit the physically expected transient behavior.

In mathematical terms, the problem at hand is to find an orbit of an associated system of four ordinary differential equations (see $(6)_{0}$ ) which connects two distinct critical points. The critical points of $(6)_{0}$ consist of two isolated critical points, $X_{L^{*}}$ and $X_{L}^{*}$, at which the mass fraction, $z$, of the unburned gas equals zero, and a curve of critical points, $C(z)$, along which $T \leqslant T_{i}$ and $z \geqslant z_{i}$ for some $z_{i}>0$. Since the gas 
is assumed to consist only of the unburned substance at $+\infty$, we seek a solution which connects $X_{L}^{*}$ at $-\infty$ to $X_{R}=C(1)$ at $+\infty$. The difficulty is that there exists a continuum of solutions which connect $X_{L}^{*}$ at $-\infty$ to critical points along $C(z)$. The "correct" solution is obtained by first showing that there exist two solutions $X_{0}(\xi)$ and $X_{1}(\xi)$ in the unstable manifold of $X_{L}^{*}$ which tend to critical points $C\left(z_{0}\right)$ and $C\left(z_{1}\right)$ along $C(z)$, with $z_{0}<1<z_{1}$. The solution with the desired limiting behavior is obtained by applying a shooting argument in a portion of the unstable manifold of $X_{L}^{*}$. Thus the main problem is to find two solutions $X_{0}(\xi)$ and $X_{1}(\xi)$ with the appropriate behavior at $+\infty$.

The location of such solutions is complicated by the degenerate character of the equations near the curve $C(z)$. However, the chemical variables (essentially) decouple from the fluid variables whenever $T \leqslant T_{i}$ (we will see that this is precisely the case along the solutions in question). The idea is to modify the kinetics $\phi(T)$ to a function $\Phi(T, z)$ when $T \leqslant T_{i}$ in such a manner that the resulting system, (6), admits precisely four hyperbolic critical points: $X_{L^{*}}, X_{L}^{*}, X_{R}$, and $X_{i}=C\left(z_{i}\right)$. It then becomes possible to bring to bear Conley's index of isolated invariant sets (cf. [1]), in the analysis of the modified flow.

(An invariant set $S$ of a flow is isolated if it is the maximal invariant set in some compact neighborhood, $N$, of itself; $N$ is called an isolating neighborhood. A compact neighborhood $N$ is therefore isolating if every solution curve through a point in $\partial N$ eventually leaves $N$ in at least one direction. The set $S(N)$ of solution curves which stay in $N$ for all time is then an isolated invariant set. A homotopy invariant associated with $S(N)$ can then be defined which furnishes some information about the structure of $S(N)$. In the present context, the index roughly plays the role of an intersection number of stable and unstable manifolds of distinct critical points; see Appendix 4 for further details.)

The unstable manifold of $X_{L}^{*}$ contains solutions which closely approximate solutions in the (one-dimensional) unstable manifold $\mathfrak{T}^{u}$ of $X_{L^{*}}$ (see Figure 17). In order to locate the solution $X_{0}(\xi)$, we therefore study the global behavior of $\Re^{u}$ with respect to the (modified) equations (6). To this end, an isolating neighborhood $N_{w}$ is constructed which contains $X_{L^{*}}$ and $X_{i}$; in this region, $z<\bar{z}$ for some $\bar{z} \in\left(z_{i}, 1\right)$. This construction requires that the viscosity coefficients satisfy certain conditions which depend on the choice of $\bar{z}$. It is then shown that $\mathfrak{R}^{u} \subset S\left(N_{w}\right)$; this follows from the "nontriviality" of the index of $S\left(N_{w}\right)$. The index is computed by exploiting its homotopy invariance; in particular, we allow the coefficients of species diffusion and heat conduction to tend to zero. The resulting system is (after a change of variables) the product of a two dimensional linear system admitting an attracting critical point, with a planar system which is similar to a qualitative model for dynamic combustion which was recently introduced by Majda [6]. Here, the index can easily be computed.

The solution $X_{1}(\xi)$ is located by showing that there is a solution of the modified equations which connects $X_{L}^{*}$ to $X_{R}$. To this end, an isolating neighborhood $N_{s}$ is constructed which contains $X_{L}^{*}$ and $X_{R}$ (but not $X_{L^{*}}$ and $X_{i}$ ). In addition, a neighborhood of the orbit $\mathfrak{R}^{u}$ must be excised from phase space. Here, essential use is made of the fact that $z<1$ along $\Re^{u}$. (If the viscosity coefficients are too large, $z$ 
will exceed unity along $\mathfrak{T}^{u}$, and this excised region can act as a "barrier" which prevents the existence of connections from $X_{L}^{*}$ to $X_{R}$.) The index of $S\left(N_{s}\right)$ is again computed by continuing the equations to Majda's model. It too turns out to be nontrivial, which forces the existence of the desired solution.

The solutions of the modified equations obtained in this manner will also be solutions of the original equations whenever $T \geqslant T_{i}$. In order to obtain the solutions $X_{0}(\xi)$ and $X_{1}(\xi)$ of the original equations, $(6)_{0}$, we solve $(6)_{0}$ in forward time, using as "initial data" the values of the solutions of the modified system obtained from index arguments at the first point at which their $T$-components equal $T_{i}$. The proof is concluded with a fairly simple shooting argument.

We remark that the conditions on the viscosity coefficients are independent of the (nonphysical) modifications introduced into the equations.

In related work, Majda and Rosales [7] and Fife [5] have shown that the full equations with certain small parameters can formally be reduced to Majda's model. This approach has the advantage of being applicable to phenomena in several space variables. However, it also requires that the shock be of sufficiently weak strength, or equivalently, that the wave velocity is only slightly larger than the sound speed of the unburned gas. The results presented here obtain for shocks of arbitrary strength. It is desirable to be able to consider strong shocks since in typical situations the differential in temperature across the shock zone will be extremely large (on the order of $1500^{\circ}$; cf. Zeldovich and Kampaneets [10, p. 72]). In addition, the width of a shock layer for strong shocks in an almost inviscid gas is on the order of the mean free path, and the validity of the macroscopic equations of hydrodynamics is questionable in the presence of such phenomena (cf. [10, p.17]). Thus, it is of interest to show that the macroscopic equations admit solutions with the "correct" qualitative behavior.

The relevance of the topological methods used here was first recognized by Conley and Smoller in connection with related questions arising in the study of nonlinear conservation laws. In particular, they have applied these methods in the study of the structure of weak shocks of quite general systems (cf. [2]), and of strong shocks for the equations of magnetohydrodynamics (cf. [3]). In these situations, it is relatively easy to find a Liapunov function for the travelling wave flow; this facilitates the construction of suitable isolating neighborhoods. The main difficulty with the inclusion of a chemical component is that a global Liapunov function does not exist, and isolating neighborhoods are more difficult to locate. A similar problem arises when dispersion terms are taken into account (cf. Smoller and Shapiro [8]).

2. The equations of reactive gas flow. In Lagrangian coordinates, the equations take the form

$$
\left\{\begin{array}{c}
\tau_{t}-u_{x}=0 \\
u_{t}+p_{x}=\left(\mu \tau^{-1} u_{x}\right)_{x}, \\
\left(e+u^{2} / 2\right)_{t}+(p u)_{x}=\left(\mu \tau^{-1} u u_{x}\right)_{x}+\left(\lambda \tau^{-1} T_{x}\right)_{x}, \\
z_{t}=-\phi(T) z+D z_{x x}
\end{array}\right.
$$


(see Majda [6]). ${ }^{2}$ Here,

$$
\begin{aligned}
\tau & =\text { specific volume } \\
u & =\text { fluid velocity in Lagrangian coordinates, } \\
T & =\text { absolute temperature } \\
p & =\text { pressure } \\
e & =\text { internal energy/unit mass of the gas mixture, } \\
z & =\text { mass fraction of the unburned gas } \\
\phi & =\text { reaction rate law. }
\end{aligned}
$$

Also, $\mu, \lambda$, and $D$ are positive coefficients representing viscosity, heat conduction, and species diffusion, respectively. Each variable is a function of $(x, t)$ where $-\infty<x<\infty$ and $t \geqslant 0$.

A. Some hypotheses. The reaction is assumed to be of the simplest form, $A \rightarrow B$, i.e., there is a single product and a single reactant. Let $z$ denote the mass fraction of the reactant; then $1-z$ is the mass fraction of the product.

The gas mixture is assumed to be polytropic. Thus, we assume that

$$
p \tau=R T,
$$

where $R$ is a positive constant which is inversely proportional to the molecular weight of the mixture. Let $e_{u}$ and $e_{b}$ be the internal energies/unit mass of the unburned and burned gas, respectively. In addition to (2), we assume that $e_{u}=c T$ $+g_{u}, e_{b}=c T+g_{b}$, where $c$ and $g_{u}\left(g_{b}\right)$ are positive constants representing the specific heat at constant volume and the energy of formation of the unburned (burned) substance. The total internal energy of the gas mixture per unit mass is therefore

$$
e=z e_{u}+(1-z) e_{b}=c T+q_{0} z+g_{b}
$$

here, $q_{0}$ is the liberated energy, which is assumed to be positive.

For more complicated reaction mechanisms, $z$ may be thought of as a "progress variable". In this case, the gas constants $R$ and $c$ will depend on $z$. Thus implicit in (2) and (3) is the assumption that variation in chemical composition has a negligible effect on the mechanical properties of the gas. Since index arguments remain valid under perturbation, the results obtained here apply if the variation of $R$ and $c$ with $z$ is sufficiently small.

If the variation in the fluid variables is small, the form (3) of the internal energy is a good approximation. However, if the variation of $T$ is large (as is the case with strong shocks), $c$ will also depend on temperature. For ideal gases, the term " $c T$ " in (3) could be replaced with an arbitrary monotone increasing function of $T$ without affecting the argument. In a similar manner the coefficients $\lambda, \mu$, and $D$ will depend on the fluid variables if the variation in the latter is large. For notational convenience, we assume that $\lambda, \mu$, and $D$ are constant. It will be evident at the end of the

\footnotetext{
${ }^{2}$ Note that there is a misprint in [6]: " $\nu_{x}$ " and " $v$ " should be interchanged in the energy balance equation.
} 
proof that $\lambda$ and $\mu$ may be allowed to be nonconstant, provided that they are majorized by small positive constants (see $\S 6$.E).

The kinetics $\phi(T)$ is assumed to be a smooth, nondecreasing nonnegative function such that $\phi(T)>0$ for $T>T_{i}$, and $\phi(T)=0$ for $T \leqslant T_{i}$; here, $T_{i}$ is the ignition temperature. The modification $\Phi(T, z)$ of $\phi$ mentioned in the introduction is a $C^{1}$ function of $(T, z)$ satisfying

$$
\Phi(T, z)= \begin{cases}\phi(T), & T \geqslant T_{i}+\eta \text { and } z \leqslant 1, \\ -(1-z)\left(T_{i}-T\right), & T \leqslant T_{i} \text { and } z \leqslant 1 .\end{cases}
$$

In addition, we choose $\Phi$ to be positive for all $z>1$. Thus, $\Phi$ changes sign in the manner indicated in Figure 1. Here, $\eta$ is small and positive. For technical reasons, we shall require that $\Phi_{T}\left(T_{i}, z\right)>0$ for all $z<1$. Since $\phi^{\prime}\left(T_{i}\right)=0$, this requires that we modify $\phi$ in the thin shaded strip in the figure so that the left and right $T$-derivatives of $\Phi$ match up at $T=T_{i}$. The main results will hold for all $\eta>0$; thus they remain valid in the limiting case where $\eta=0$ (cf. §6.C). (We could also assume that $\phi$ is Lipschitz continuous at $T=T_{i}$ with positive right-hand derivative at $T_{i}$. The waves for smooth $\phi$ are then obtained as limits of those obtained for Lipschitz $\phi$ as $D^{+} \phi\left(T_{i}\right)$ tends to zero.)

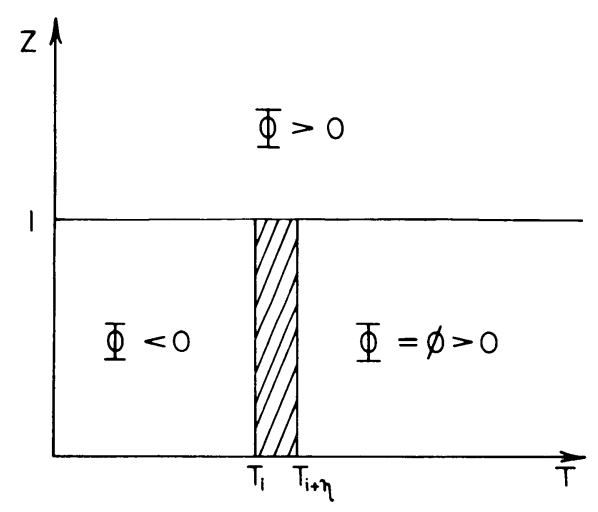

FIGURE 1

We shall also have to modify the species diffusion coefficient $D$ to a function $d(T)$ when $T<T_{i}$ (see $\mathrm{D}$, below).

B. The travelling wave equations. A travelling wave solution of (1) depends on the single quantity $\xi=x-\sigma t$, where $\sigma$ (the wave velocity) is a positive constant. (With $\sigma>0$, the unburned gas is on the right at $\xi=+\infty$ and the burned gas is on the left at $\xi=-\infty$, since with positive $\sigma$ the wave front moves from left to right.) Such solutions satisfy the system of o.d.e.'s

$$
\left\{\begin{array}{c}
-\sigma \tau^{\prime}=u^{\prime}, \\
-\sigma u^{\prime}+p^{\prime}=\left(\mu \tau^{-1} u^{\prime}\right)^{\prime}, \\
-\sigma\left(e+u^{2} / 2\right)^{\prime}+(p u)^{\prime}=\left(\mu \tau^{-1} u u^{\prime}\right)^{\prime}+\left(\lambda \tau^{-1} T^{\prime}\right)^{\prime} \\
-\sigma z^{\prime}=-\Phi z+d z^{\prime \prime}
\end{array}\right.
$$


where "prime" is $d / d \xi$. Suppose that a solution $(\tau, u, T, z)$ of (4) tends to a limit $\left(\tau_{R}, u_{R}, T_{R}, 1\right)$ as $\xi \rightarrow+\infty$, where $T_{R}<T_{i}$ and $\tau_{R}>0$. It follows that $\tau^{\prime}, T^{\prime}$, and $u^{\prime}$ tend to zero as $\xi \rightarrow+\infty$. The first three equations in (4) can be integrated from $\xi$ to $+\infty$ to obtain

$$
\begin{gathered}
-\sigma\left(\tau-\tau_{R}\right)=u-u_{R}, \\
\tau\left[-\sigma\left(u-u_{R}\right)+p-p_{R}\right]=\mu u^{\prime}, \\
\tau\left[-\sigma\left(e-e_{R}\right)-\sigma\left(u^{2}-u_{R}^{2}\right) / 2+p u-p_{R} u_{R}\right]=\mu u u^{\prime}+\lambda T^{\prime} ;
\end{gathered}
$$

here $p_{R}=R T_{R} \tau_{R}^{-1}$ and $e_{R}=c T_{R}+g_{u}$ are obtained from (2) and (3). It follows that (5a) can be used to eliminate $u$ and $u_{R}$ from the remaining equations. If we introduce a new variable $w=z^{\prime}$, the final equations can be written as the four dimensional first order system

$$
\left\{\begin{array}{c}
\mu \tau^{\prime}=-\sigma^{-1}\left[\sigma^{2} \tau^{2}-A \tau+R T\right] \equiv F_{1}(\tau, T), \\
\lambda T^{\prime}=\sigma \tau\left[\sigma^{2} \tau^{2} / 2-A \tau+B-q_{0}(z-1)-c T\right] \equiv \sigma \tau F_{2}(\tau, T, z), \\
z^{\prime}=w \\
D w^{\prime}=-\sigma w+\phi(T) z
\end{array}\right.
$$

where $A=\sigma^{2} \tau_{R}^{2}+p_{R}$ and $B=\sigma^{2} \tau_{R}^{2} / 2+\tau_{R} p_{R}+c T_{R}$ are positive constants. The modified equations take the form

$$
\left\{\begin{array}{c}
\mu \tau^{\prime}=F_{1}(\tau, T) \\
\lambda T^{\prime}=\sigma \tau F_{2}(\tau, T, z) \\
z^{\prime}=w \\
d(T) w^{\prime}=-\sigma w+\Phi(T, z) z
\end{array}\right.
$$

The details are given in Appendix 1. In the sequel, the vector of dependent variables will be denoted by $X=(\tau, T, z, w)$.

C. The critical points of $(6)_{0}$ and (6). By construction, it is clear that $X_{R}=$ $\left(\tau_{R}, T_{R}, 1,0\right)$ is a critical point of $(6)_{0}$ and (6). The claim is that for each $\left(\sigma, q_{0}\right)$ in the shaded region of Figure 0 there are precisely three other critical points of $(6)$, which will be denoted by

$$
X_{L^{*}}=\left(\tau_{L^{*}}, T_{L^{*}}, 0,0\right), \quad X_{L}^{*}=\left(\tau_{L}^{*}, T_{L}^{*}, 0,0\right), \quad X_{i}=\left(\tau_{i}, T_{i}, z_{i}, 0\right) .
$$

Here, $T_{i}$ is the ignition temperature, $T_{R}<T_{i}<T_{L^{*}}<T_{L}^{*}$, and $0<z_{i}<1$. (There are other critical points with $\tau=T=0$, but these will not enter into the discussion.) The proof involves an analysis of the Rankine-Hugoniot relations associated with (5), and a comparison of these conditions with the additional restriction that $w=\Phi(T, z) z=0$ at critical points. The details are given in Appendix 2. As a consequence of this analysis, it follows that the curves $F_{1}=0$ and $F_{2}=0$ in the $(\tau, T)$ plane have the aspect indicated in Figure 2, for various values of $z$.

The analysis is similar for the original system, $(6)_{0}$. However, since $\phi(T)=0$ for $T \leqslant T_{i}$, the right-hand intersection point of $F_{1}=0$ with $F_{2}=0$ will yield a critical point for each $z \geqslant z_{i}$. For each such $z$, we denote the associated critical point by $C(z)$. 


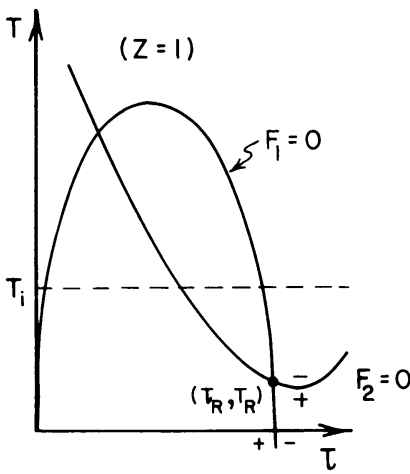

(a)

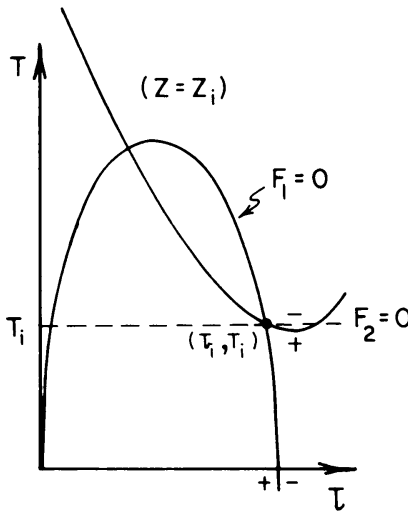

(b)

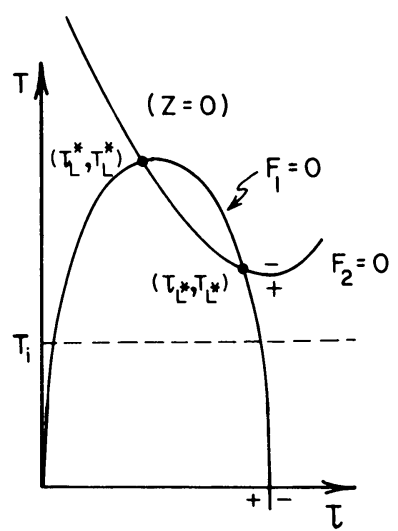

(c)

FIGURE 2

D. Modification of $D$. The modified kinetic $\Phi$ was introduced in order to "push" solutions away from the plane $z=1, w=0$, whenever $T<T_{i}$. In order that $\Phi$ have the desired effect, it will be necessary to modify $D$ to a function $d=d(T)$ with the following properties: $d(T)=D$ for $T \geqslant T_{i}, d(T)=\varepsilon$ for $T \leqslant T_{i}-\rho$, and $d(T)$ is a smooth, nondecreasing function of $T$ for all $T$. Here, $\varepsilon<D$ and $\rho=\rho(\varepsilon)$ are small positive constants to be determined later.

E. The main result. The following theorem will be proved in $\S \S 3-6$.

THEOREM 2.1. Suppose that the hypotheses of $§ 2$.A hold and that $\left(\sigma, q_{0}\right)$ lies in the shaded region of Figure 0 . Suppose also that $\lambda, \mu$, and $D$ satisfy the following conditions:

$$
\begin{gathered}
\left(\lambda^{2}+\mu^{2}\right)^{1 / 2}<\min (a, b)^{2} /\left(8 q_{0} \tau_{2} K \Delta\right), \\
\mu<c a / q_{0} L, \\
D<\min (\lambda \Gamma,(1-\bar{z}) \sigma / L) .
\end{gathered}
$$

Then there exist solutions $X_{w}(\xi)$ and $X_{s}(\xi)$ of $(6)_{0}$ which satisfy the boundary conditions

$$
\begin{array}{ll}
\lim _{\xi \rightarrow-\infty} X_{w}(\xi)=X_{L^{*}}, & \lim _{\xi \rightarrow+\infty} X_{w}(\xi)=C\left(z_{*}\right), \\
\lim _{\xi \rightarrow-\infty} X_{s}(\xi)=X_{L}^{*}, & \lim _{\xi \rightarrow+\infty} X_{s}(\xi)=X_{R},
\end{array}
$$

where $C\left(z_{*}\right)$ is a critical point in $C(z)$ with $z_{*}<1$.

The constants appearing in conditions (7)-(9) depend on certain geometric properties of the vector field on the right-hand side of $(6)_{0}$; they will be defined later (see (10), (11), (17), and (24)). For the moment, it suffices to remark that their role is to ensure that the $z$-component of $X_{w}$ is always less than unity.

The solution $X_{s}(\xi)$ is called a strong detonation; $X_{w}(\xi)$ is called a weak detonation. Since $z=1$ at $+\infty$ the former solution is the correct, physically observed solution. In particular, if the viscosity coefficients are zero and the reaction proceeds infinitely fast, $X_{s}$ corresponds to the "entropy" solution of the associated conservation laws. 
However, for a certain measure zero set of $\left(\sigma, q_{0}\right), X_{w}$ is the "correct" solution. This can be seen by noting that $(6)_{0}$ is linear with respect to $(z, w)$. Thus, if $X=(\tau, T, z, w)$ is a solution of $(6)_{0}$, then $(\tau, T, c z, c w)$ is also a solution of $(6)_{0}$, but with a different liberated energy, namely, $c^{-1} q_{0}$. If $X=X_{w}$ and $c^{-1}=z_{*}$, then for this liberated energy, $X_{w}$ tends to $X_{R}$ at $+\infty$. For liberated energies less than $z_{*} q_{0}$, the limiting value of $z$ along $X_{w}$ will exceed unity. It is reasonable to expect that under such conditions, no detonation profile exists, and that there is a transfer of stability to a slower, deflagration profile.

The remainder of the paper is devoted to the proof of Theorem 2.1. In $\S 3$, isolating neighborhoods $N_{w}$ and $N_{s}$ are constructed for the modified flow, (6). In $\S \S 4$ and 5 , the (modified) flow is continued to a simpler set of equations where the indices of the maximal invariant sets $S\left(N_{w}\right)$ and $S\left(N_{s}\right)$ in $N_{w}$ and $N_{s}$ are easily computed. The nontriviality of these indices is then used in $\$ 6$ to obtain the solutions $X_{w}$ and $X_{s}$ of $(6)_{0}$.

3. Isolating neighborhoods for the modified flow. In this section, isolating neighborhoods $N_{w}$ and $N_{s}$ with respect to (6) are constructed which contain exactly two critical points, namely $X_{L^{*}}, X_{i}$, and $X_{L}^{*}, X_{R}$, respectively. As explained in the introduction, the aim is to recover information about certain solutions of the unmodified flow, (6) $)_{0}$, from the behavior of solutions in $S\left(N_{w}\right)$ and $S\left(N_{s}\right)$.

A. Constructiion of $N_{w}$. Let $\bar{z} \in\left(z_{i}, 1\right)$ be given. Conditions on $\mu, \lambda$, and $D$ will be determined which imply that $\bar{z}$ is an upper bound for $z$ along the unstable manifold of $X_{L^{*}}$.

Let $\bar{F}_{2}(\tau, T)=F_{2}(\tau, T, \bar{z})$, and let $B$ be the rectangle in the $(\tau, T)$ plane indicated in Figure 3. Here, $r>0$ is a small positive constant. Note that the vector field $\left(F_{1}, \sigma \tau F_{2}\right)$ points into the interior of $B$ along $\partial B$ for each $z \in[-r, \bar{z}]$.

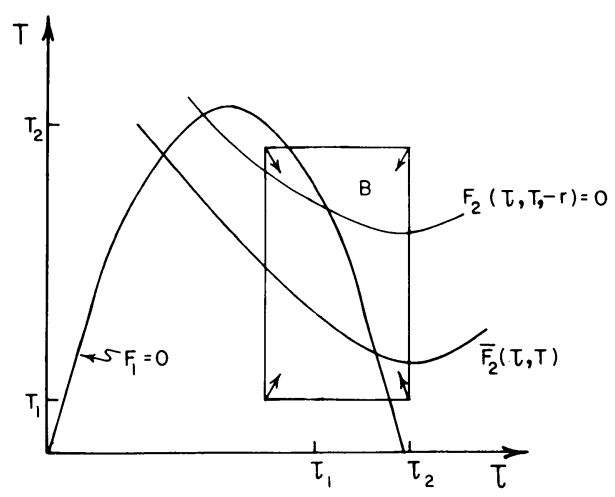

Figure 3

Let $K>0$ be such that $|\Phi(T, z) z|<K$ for $T \in\left[T_{1}, T_{2}\right]$ and $z \in[-r, \bar{z}]$, and let $L=\sigma^{-1} K$. Finally, let $A_{r}$ be a small closed ball of radius $r$ and center $X_{L^{*}}$.

The neighborhood $N_{w}$ is defined by

$$
N_{w}=B \times\{(z, w): 0 \leqslant z \leqslant \bar{z},|w| \leqslant L\} \cup A_{r}=N_{1} \cup A_{r} .
$$


THEOREM 3.1. Suppose that $\lambda$ and $\mu$ satisfy (7) and (8) of Theorem 2.1. Then $N_{w}$ is an isolating neighborhood of (6), provided that $\varepsilon$ and $\rho$ are sufficiently small (see §2.D).

Proof. Let $X_{0}=\left(\tau_{0}, T_{0}, z_{0}, w_{0}\right) \in \partial N_{w}$. It must be shown that the solution curve through $X_{0}$ at $\xi=0$ leaves $N_{w}$ in at least one time direction. At many points, the curve will be transverse to $\partial N_{w}$ so that the solution immediately leaves $N_{w}$ in one direction. At other points, the solution is tangent to $\partial N_{w}$, and the possibility arises that the solution stays in $N_{w}$ in both directions. In this case, the solution must be followed in either forward or backward time to a point where it leaves $N_{w}$.

Various types of boundary points are treated in each of the following lemmas. Together, they include all points in $\partial N_{w}$.

LEMMA 3.2. Suppose that
(a) $\left(\tau_{0}, T_{0}\right) \in \partial B$,
(b) $z_{0}=0$ and $X_{0} \notin A_{r}$,
(c) $X_{0} \in \partial A_{r} \backslash N_{1}$,
(d) $X_{0} \in \partial A_{r} \cap \partial N_{1}$,or
(e) $\left|w_{0}\right|=L$.

Then in each case, either the solution curve through $X_{0}$ eventually leaves $N_{w}$ or $X_{0}$ is an interior point of $N_{w}$.

Proof. (a) Since the vector field $\left(F_{1}, \sigma \tau F_{2}\right)$ points into the interior of $B$ along $\partial B$ for each $z \in[-r, \bar{z}]$, it follows that the $(\tau, T)$ components leave $B$ in backward time. The solution therefore leaves $N_{w}$.

(b) If $z_{0}=0$, then $z$ either assumes negative values in one direction, or $z$ remains nonnegative. In the former case, the solution leaves $N_{w}$, since $X_{0} \notin A_{r}$. In the latter case, $w_{0}=0$. Since $w=z=0$ is an invariant manifold of (6), it follows that $w=z=0$ along the entire solution. Since $X_{0} \notin A_{r}$, the solution is not the critical point at $X_{L^{*}}$. It follows that the $(\tau, T)$ components leave $B$ in backward time.

(c) If $X_{0} \in \partial A_{r} \backslash N_{1}$, then $z_{0}<0$, provided that $r$ is small enough that the projection of $A_{r}$ on the $(\tau, T)$ plane is contained in the interior of $B$. Suppose first that $w_{0}>0$. Then $z$ decreases in backward time so that the orbit must remain in $A_{r}$ in this direction for as long as $w$ remains positive. Suppose that $w(\xi)=0$ for some $\xi<0$. At this point, $D w^{\prime}=\Phi z<0$, since $\Phi>0$ in $A_{r}$ and $z<0$. Thus $z^{\prime \prime}<0$, so that $z$ has a strict local maximum, which is impossible. It follows that $w(\xi)>0$ for all $\xi \leqslant 0$. If the solution were to remain in $N_{w}$ (and hence, $A_{r}$ ) in this direction, $z$ would converge to a negative limit. It is easily seen that each of the other components would also have to converge to limits in backward time. The limiting value of the solution would therefore have to be a critical point. Since there are no critical points in $A_{r}$ with $z<0$, the solution eventually leaves $A_{r}$ while $z<0$, and so, leaves $N_{w}$.

If $w_{0}<0$, a similar argument shows that the solution leaves $N_{w}$ in forward time. Finally, if $w_{0}=0, D z^{\prime \prime}(0)=\Phi z<0$, so that $z$ has a strict local maximum at $z_{0}$. It follows that the solution leaves $N_{w}$ in both directions.

(d) If $X_{0} \in \partial A_{r} \cap \partial N_{1}$, then $z_{0}=0$. If $w_{0} \neq 0$, the solution enters $\{z<0\}$ in one direction and (c) applies. If $w_{0}=0$, then $z=w=0$ along the entire solution, and (b) applies. 
(e) If $\left|w_{0}\right|=L$, then $d w^{\prime}(0)= \pm \sigma L+\Phi z_{0} \neq 0$. It follows that $|w|>L$ in one direction, and the solution leaves $N_{w}$.

The remaining case is when $z_{0}=\bar{z}$. If $w_{0} \neq 0$, then $z>\bar{z}$ in one direction, and the solution leaves $N_{w}$. If $w_{0}=0$ and $T_{0}>T_{i}$, then $D w^{\prime}=\Phi\left(T_{0}, \bar{z}\right) \bar{z}>0$, since $\Phi>0$. Thus $z$ has a strict local minimum at $\bar{z}$ and the solution leaves $N_{w}$ in both directions.

The next two lemmas treat the remaining case, namely $z_{0}=\bar{z}, w_{0}=0$, and $T_{0} \leqslant T_{i}$. The following notation will be needed.

Let $\bar{T}$ be the value of $T$ at the intersection of $\bar{F}_{2}=0$ with $F_{1}=0$; since $\bar{z}>z_{i}$, it follows that $\bar{T}<T_{i}$. Select $T_{*} \in\left(\bar{T}, T_{i}\right)$, and let $l$ be the line segment indicated in Figure 4. Finally, let $S_{L}\left(S_{R}\right)$ be the points in $B$ with $T \geqslant T_{*}$ which lie to the left (right) of $l$, and let $S$ be the shaded region in Figure 4.

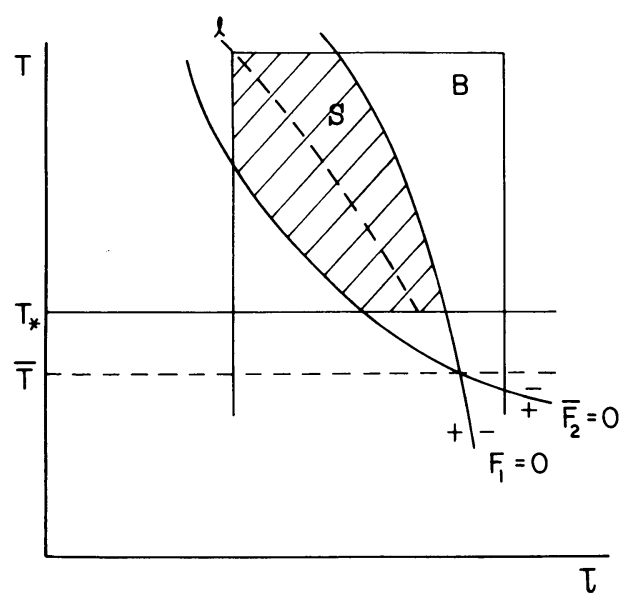

FIGURE 4

Let $a, b$, and $c$ be defined by

$$
\left\{\begin{array}{c}
a=\min \left(\min \left\{F_{1}(\tau, T):(\tau, T) \in S_{L}\right\}, a^{\prime}, a^{\prime \prime}\right), \\
b=\min \left(\min \left\{-\sigma \tau \bar{F}_{2}(\tau, T):(\tau, T) \in S_{R}\right\}, b^{\prime \prime}\right), \\
c=\min \left(\min \left\{-\sigma \tau \partial F_{2} / \partial \tau(\tau, T, z): F_{2}(\tau, T, z)=0,(\tau, T) \in B\right\}, c^{\prime}\right)
\end{array}\right.
$$

here, $a^{\prime}, a^{\prime \prime}, b^{\prime \prime}$, and $c^{\prime}$ are similarly defined positive constants that will be needed in the construction of $N_{s}$ (see (17) and §4.C). From Figure 4, it is evident that $a$ and $b$ are both positive. Moreover, $\partial F_{2} / \partial \tau<0$ along $F_{2}=0$; thus $c$ is positive also.

Lemma 3.3. Suppose that $z_{0}=\bar{z}, w_{0}=0$, and $T_{*} \leqslant T_{0} \leqslant T_{i}$, and that $\lambda$ and $\mu$ are chosen small enough that (7) and (8) hold. Then the solution curve through $X_{0}$ leaves $N_{w}$.

Proof. Let

$$
\left\{\begin{array}{c}
\Delta=T_{2}-T_{*}+\tau_{2}-\tau_{1}, \\
\xi_{0}=-4\left(\lambda^{2}+\mu^{2}\right)^{1 / 2} \Delta / \min (a, b),
\end{array}\right.
$$


where $\tau_{1}, \tau_{2}$ and $T_{2}$ are as in Figure 3. There are three cases; together, they include all possibilities.

(i) $\left(\tau_{0}, T_{0}\right) \in S$, and $\tau^{\prime}(\xi)>0, T^{\prime}(\xi)<0$ for all $\xi \in\left[\xi_{0} / 2,0\right]$;

(ii) $T^{\prime}(\xi) \geqslant 0$ for some $\xi \in\left[\xi_{0} / 2,0\right]$;

(iii) $\tau^{\prime}(\xi) \leqslant 0$ for some $\xi \in\left[\xi_{0} / 2,0\right]$.

(i) Since $F_{2}(\tau, T, z)=0$ lies above $\bar{F}_{2}(\tau, T)=0$ for each $z<\bar{z}$, the assumption in (i) implies that $(\tau, T)$ lies in the region $S$ in Figure 4 for $\xi \in\left[\xi_{0} / 2,0\right]$. For such $\xi$, it follows that

$$
\begin{aligned}
\tau^{\prime}-T^{\prime} & =\left|\tau^{\prime}\right|+\left|T^{\prime}\right| \\
& \geqslant\left(\lambda^{2}+\mu^{2}\right)^{1 / 2}\left(\mu\left|\tau^{\prime}\right|+\lambda\left|T^{\prime}\right|\right) \\
& \geqslant\left(\lambda^{2}+\mu^{2}\right)^{-1 / 2}\left(\min (a, b)-q_{0} \sigma \tau_{2}|z-\bar{z}|\right) \\
& \geqslant\left(\lambda^{2}+\mu^{2}\right)^{-1 / 2}\left(\min (a, b)-q_{0} \tau_{2} K|\xi|\right),
\end{aligned}
$$

since $z(0)=\bar{z}$, and $L$, the bound for $z^{\prime}$ in $N_{w}$, equals $\sigma^{-1} K$. From (7) and (11), it follows that $q_{0} \tau_{2} K|\xi|<\min (a, b) / 2$ for each $\xi \in\left[\xi_{0}, 0\right]$. Integration of the above inequality for $\tau^{\prime}-T^{\prime}$ from $\xi_{0} / 2$ to zero (together with (7) and (11)) yields the estimate

$$
\begin{aligned}
\tau_{0}-\tau\left(\xi_{0} / 2\right)+T\left(\xi_{0} / 2\right)-T_{0} & >\left|\xi_{0} / 2\right|\left(\lambda^{2}+\mu^{2}\right)^{-1 / 2}\left[\min (a, b)-q_{0} \tau_{2} K\left|\xi_{0} / 4\right|\right] \\
& >\left|\xi_{0} / 2\right|\left(\lambda^{2}+\mu^{2}\right)^{-1 / 2}[\min (a, b)-\min (a, b) / 8] \\
& >(2 \Delta / \min (a, b))(7 \min (a, b) / 8)>\Delta .
\end{aligned}
$$

Since $T_{0} \geqslant T_{*}$ and $\tau_{0}<\tau_{2}$, it follows that either $T\left(\xi_{0} / 2\right)<T_{2}$ or $\tau\left(\xi_{0} / 2\right)<\tau_{1}$. In either case, the solution leaves $N_{w}$.

(ii) Now suppose that $T^{\prime}\left(\xi_{1}\right) \geqslant 0$ for some $\xi_{1} \in\left[\xi_{0} / 2,0\right]$. It will first be shown that if $T^{\prime}(\bar{\xi})=0$ for any $\bar{\xi} \in\left[\xi_{0}, 0\right]$, then $(\tau, T)$ lies on the left side of $l$ at $\bar{\xi}$. To this end, note that at $\bar{\xi}$,

$$
0=\left[F_{2}(\tau, T, z)-\bar{F}_{2}(\tau, T)\right]+\bar{F}_{2}(\tau, T)=q_{0}(z-\bar{z})+\bar{F}_{2}(\tau, T),
$$

so that

$$
\left|\sigma \tau \bar{F}_{2}(\tau, T)\right|<q_{0} \tau_{2} K|\bar{\xi}|<\min (a, b) / 2<b .
$$

Since $\sigma \tau \bar{F}_{2}<-b$ to the right of $l$, the claim follows.

Now suppose that $\mu$ is so small that (8) holds. If $T^{\prime}(\bar{\xi})=0$, it follows that

$$
\lambda T^{\prime \prime}(\bar{\xi})=\sigma \tau\left(\partial F_{2} / \partial \tau \tau^{\prime}-q_{0} z^{\prime}\right)<\sigma \tau\left(-c a / \mu+q_{0} L\right)<0 .
$$

Thus $T$ has a local maximum at every critical point $\bar{\xi}$ of $T$ in the interval $\left[\xi_{0}, 0\right]$. It follows that $T$ has at most one critical point on this interval.

Suppose first that $T^{\prime}(\bar{\xi})=0$ for some $\bar{\xi} \in\left[\xi_{0}, 0\right]$. From the above it follows that $T^{\prime}(\xi)>0$ for $\xi \in\left[\xi_{0}, \bar{\xi}\right]$. The claim is that this monotonicity persists for all $\xi<\xi_{0}$. First note that $\tau^{\prime}(\bar{\xi})>0$, since $(\tau, T) \in S_{L}$ at $\bar{\xi}$. Since $F_{1}=0$ has negative slope in $B$, it follows that $\tau^{\prime}(\xi)$ remains positive for $\xi<\bar{\xi}$ for at least as long as $T^{\prime}$ remains positive. Suppose that $T^{\prime}\left(\xi^{*}\right)=0$ for some $\xi^{*}<\bar{\xi}$. If $F_{1}(\tau, T)>a$ at $\xi^{*}$, the estimate (13) yields a contradiction. The problem is that now $T\left(\xi^{*}\right)$ may be less than $T_{*}$. 
However, from the preceding remarks it follows that $\left(\tau\left(\xi^{*}\right), T_{*}\right) \in S_{L}$. If $T\left(\xi^{*}\right)<T_{*}$, we have that $F_{1}\left(\tau\left(\xi^{*}\right), T\left(\xi^{*}\right)\right)>F_{1}\left(\tau\left(\xi^{*}\right), T_{*}\right)>a$, since $\partial F_{1} / \partial T<0$. Thus (13) holds at $\xi=\xi^{*}$. It follows that $\tau$ and $T$ are both monotone decreasing in backward time. Since there are no critical points in this region, the solution must eventually leave $N_{w}$.

If $T^{\prime}(0)>0$, the result follows from a similar, simpler argument.

(iii) Suppose now that $\tau^{\prime}(\bar{\xi}) \leqslant 0$ for some $\bar{\xi} \in\left[\xi_{0} / 2,0\right]$. Suppose also that $T^{\prime}(\xi)<0$ on $\left[\xi_{0} / 2,0\right]$; otherwise case (ii) applies. Assume that $\bar{\xi}$ is the largest nonpositive $\xi$ with $\tau^{\prime}(\xi) \leqslant 0$. Thus for $\xi<\bar{\xi},(\tau, T)$ moves into the region above $F_{1}=0$.

Note that $T^{\prime}<0$ on the entire interval $\left[\xi_{0}, 0\right]$, since the estimate (12) shows that $T$ could only have a critical point when $(\tau, T) \in S_{L}$, and for such $\xi,(\tau, T)$ lies above $F_{1}=0$, and hence, in $S_{R}$.

The claim is that $T\left(\xi_{0}\right)>T_{2}$. Since $(\tau, T) \in S_{R}$ for $\xi \in\left[\xi_{0}, \xi_{1}\right]$, it follows that $-\lambda T^{\prime}>b-q_{0} \tau_{2} K\left|\xi-\xi_{1}\right|>0$. Integrate this inequality from $\xi_{0}$ to $\xi_{1}$ to obtain

$$
T\left(\xi_{0}\right)-T\left(\xi_{1}\right)<\lambda^{-1}\left|\xi_{0}-\xi_{1}\right|\left(b-q_{0} \tau_{2} K\left|\xi_{0}-\xi_{1}\right| / 2\right) \text {. }
$$

Since $\left|\xi_{0}-\xi_{1}\right|>\left|\xi_{0} / 2\right|$, it follows from (7) and (11) that $T\left(\xi_{0}\right)-T\left(\xi_{1}\right)>\Delta>T_{2}$ $-T_{*}$. This completes the proof, since $T\left(\xi_{1}\right)>T_{*}$.

Lemma 3.4. Suppose that $z_{0}=\bar{z}, w_{0}=0$, and that $T_{0} \leqslant T_{*}$. Then the solution through $X_{0}$ leaves $N_{w}$, provided that $\varepsilon$ and $\rho$ are sufficiently small (cf. §2.D).

Proof. Let $M$ be an upper bound for $\left|\sigma \tau F_{2}\right|$ in $N_{w}$. Then $\left|T(\xi)-T_{0}\right| \leqslant \lambda^{-1} M|\xi|$. Let $\xi_{1}<0$ be such that $\lambda^{-1} M\left|\xi_{1}\right|=\left(T_{i}-T_{*}\right) / 2$. Since $T_{0} \leqslant T_{*}$, it follows that $T\left(\xi_{1}\right)<\left(T_{i}+T_{*}\right) / 2<T_{i}$. Let $\rho$ be so small that $\left(T_{i}+T_{*}\right) / 2<T_{i}-\rho$. It follows that $d(T)=\varepsilon$ for $\xi \in\left[\xi_{1}, 0\right]$ (cf. $\left.\S 2 . D\right)$.

Define a new variable $\pi(\xi)$ by

$$
\pi=\varepsilon w^{\prime}=-\sigma w+h(T, z),
$$

where $h(T, z)=\Phi(T, z) z$. Then $\pi$ satisfies the equation

$$
\begin{aligned}
\pi^{\prime} & =-\sigma w^{\prime}+d h \cdot\left(T^{\prime}, w\right)=-\sigma \varepsilon^{-1} \pi+d h \cdot\left(\lambda^{-1} \sigma \tau F_{2},(h-\pi) / \sigma\right) \\
& =\left(-\sigma \varepsilon^{-1}-h_{z} \sigma^{-1}\right) \pi+h_{T} \lambda^{-1} \sigma \tau F_{2}+h_{z} h / \sigma .
\end{aligned}
$$

Note that if the solution lies in $S\left(N_{w}\right)$, then $|\pi| \leqslant \sigma L+K=2 K$ for all $\xi$. We shall use (15) to show that $\left|\pi\left(\xi_{1}\right)\right|>2 K$, provided that $\varepsilon$ is sufficiently small.

First note that

$$
\pi(0)=\Phi\left(T_{0}, \bar{z}\right) \bar{z}<-\left(T_{i}-T_{*}\right)(1-\bar{z}) \bar{z}<0 .
$$

Let $P$ be a bound for $\left|h_{T} \lambda^{-1} \sigma \tau F_{2}\right|+\left|h_{z} h / \sigma\right|+\left|h_{z} / \sigma\right|$ in $N_{w}$, and let $Q=-\pi(0)$. Suppose that

$$
\varepsilon<\sigma Q /\left[P+Q P+(2 K+Q)\left|\xi_{1}\right|^{-1}\right],
$$

where $\left|\xi_{1}\right|=\left(T_{i}-T_{*}\right) \lambda /(2 M)$. It follows that $\pi^{\prime}(0)>(2 K+Q)\left|\xi_{1}\right|^{-1}>0$, so that $\pi(\xi)<\pi(0)$ for $\xi<0$. Thus $-\pi(\xi)>Q$ for all $\xi \in\left[\xi_{1}, 0\right]$, and it follows that $\pi^{\prime}(\xi)>(2 K+Q)\left|\xi_{1}\right|^{-1}$ on this interval. We therefore have that $\pi\left(\xi_{1}\right)>\pi(0)+2 K$ $+Q=2 K$; thus the solution cannot remain in $N_{w}$. 
This completes the proof of Theorem 3.1. Note that as $\bar{z}$ approaches $z_{i}, T_{*}$ approaches $T_{i}$ and the constants $a$ and $b$ appearing in (7) and (8) will approach zero. Hence the conditions on $\mu$ and $\lambda$ become more restrictive as $\bar{z} \rightarrow z_{i}$.

B. Construction of $N_{s}$. An isolating neighborhood containing $X_{L}^{*}$ and $X_{R}$ and excluding $X_{L^{*}}$ and $X_{i}$ will now be constructed. This will depend on several results which will be proved later, namely, that $X_{i}$ is an attractor for (6), and $X_{L^{*}}$ is a saddle with a one-dimensional unstable manifold. The portion of this manifold in the region $\{z>0\}$ will be denoted by $\Re^{u}$. It will also be shown that $\Re^{u}$ lies in $S\left(N_{w}\right)$, and hence, $z<1$ along this solution.

As a first approximation to $N_{s}$, consider the union $N$ of two regions $N_{1}$ and $N_{2}$, which are defined as follows. Let $\delta$ be small and positive; in particular, suppose that $0<\delta<1-\bar{z}$. From Figure 5, it is clear that there exists a rectangle $C=\left[0, \tau_{2}\right] \times$ $\left[T_{1}, T_{2}\right]$ such that $\left(F_{1}, \sigma \tau F_{2}\right)$ is transverse to $\partial C$ along $\partial C$, for all $z \in[-\delta, 1+\delta]$. Let $C_{1}=\left[0, \tau_{2}\right] \times\left[T_{i}, T_{2}\right]$, and define

$$
\begin{aligned}
& N_{1}=C_{1} \times\{(z, w): 0 \leqslant z \leqslant 1+\delta, 0 \leqslant w \leqslant L\}, \\
& N_{2}=C \times\{(z, w):|1-z| \leqslant \delta,|w| \leqslant L\} .
\end{aligned}
$$

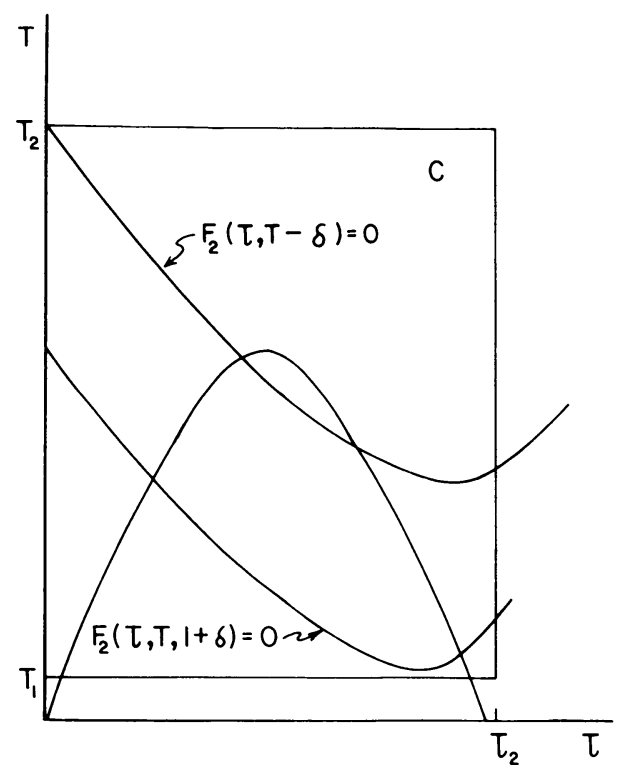

FIGURE 5

The following theorem shows that $S\left(N_{1} \cup N_{2}\right) \cap \partial\left(N_{1} \cap N_{2}\right)$ consists of certain distinguished orbits. An isolating neighborhood will finally be determined by excising suitable regions from $N_{1} \cup N_{2}$ which excludes these solutions; in particular, the excision is performed in such a manner that the maximal invariant set in this new, smaller neighborhood avoids all boundary points of the excised regions.

THEOREM 3.5. Let $N=N_{1} \cup N_{2}$, and suppose that (8) holds. Then for sufficiently small $\varepsilon$ and $\rho=\rho(\varepsilon), S(N) \cap \partial N$ consists of the critical points at $X_{L^{*}}, X_{L}^{*}$, and $X_{i}$, together with the orbit along which $z \equiv w \equiv 0$ which connects $X_{L^{*}}$ to $X_{L}^{*}$. 
(The existence of this connecting orbit is easily obtained from two-dimensional phase plane arguments; see Figure 6.)

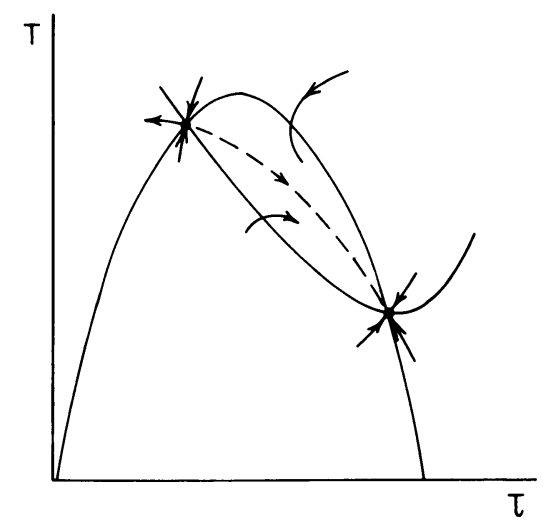

Figure 6

Proof. As before, let $X_{0} \in \partial N$ and suppose that a solution of (6) hits $X_{0}$ when $\xi=0$. The following cases will be considered separately in a sequence of lemmas:

(i) $\left|w_{0}\right|=L, z_{0}=1+\delta, \tau_{0}=0$ or $\tau_{2}$, or $T_{0}=T_{1}$ or $T_{2}$;

(ii) $z_{0}=0$, or $w_{0}=0$ and $z_{0} \neq 1-\delta$;

(iii) $T_{0}=T_{i}$ and $z_{0} \neq 1-\delta$;

(iv) $z_{0}=1-\delta$.

In each successive case, it will be assumed that the conditions in the preceding cases do not hold.

LemMa 3.6. Suppose that $X_{0}$ satisfies at least one of the conditions in (i). Then the solution through $X_{0}$ leaves $N$.

Proof. If $\left|w_{0}\right|=L$, then (as in (e) of Lemma 3.2) $d w^{\prime}(0) \neq 0$, so that $|w|>L$ in one direction.

If $z_{0}=1+\delta$ and $w_{0} \neq 0$, then $z>1+\delta$ in one direction. If $w_{0}=0$, then $d w^{\prime}(0)>0$, since $\Phi>0$ for $z=1+\delta$. Thus $z>1+\delta$ in both directions.

Finally, suppose that $\tau_{0}=0$ or $\tau_{2}$, or that $T_{0}=T_{1}$ or $T_{2}$. Since $\left(F_{1}, \sigma \tau F_{2}\right)$ is transverse to $\partial C$ along $\partial C,(\tau, T)$ leaves $C$ in one direction.

Note that the proof for $z_{0}=1+\delta$ holds for all $\delta>0$. It follows that $z \leqslant 1$ along solutions in $S(N)$.

LEMMA 3.7. Suppose that $X_{0} \in \partial N$ satisfies one of the conditions in (ii) and none of the conditions in (i). Then the solution through $X_{0}$ is either one of the orbits in the statement of Theorem 3.5, or it leaves $N$ in finite time.

Proof. Suppose first that $z_{0}=0$. If $w_{0} \neq 0$, then $z<0$ in one direction, and the solution leaves $N$. If $w_{0}=0$, then $w \equiv z \equiv 0$ along the orbit, and the $(\tau, T)$ flow is as depicted in Figure 6. The solution therefore either leaves $N$, or it is $X_{L^{*}}, X_{L}^{*}$, or their connecting orbit. 
Now suppose that $w_{0}=0$ and $z \neq 1-\delta$. If $1-\delta<z_{0}<1+\delta$, then $X_{0}$ is an interior point of $N_{2}$, and hence of $N$. Suppose then that $0<z_{0}<1-\delta$. In this case, $X_{0} \in N_{1}$ so that $T_{i} \leqslant T_{0}<T_{2}$. If $T_{0}>T_{i}$, then $d w^{\prime}(0)=\Phi z_{0}>0$, so that $w$ assumes negative values in backward time while $z<1-\delta$. Such solutions leave $N$.

The remaining case is when $w_{0}=0,0<z_{0}<1-\delta$, and $T_{0}=T_{i}$. If $T^{\prime} \neq 0$, then the solution enters the region $\left\{z<1-\delta, T<T_{i}\right\}$ in one direction, and therefore leaves $N$. Suppose then that $T^{\prime}(0)=0$. Note that $d(T)=D$ for $T>T_{i}$ and sufficiently near $T_{i}$, so that $d^{\prime}\left(T_{i}\right)=d^{\prime \prime}\left(T_{i}\right)=0$. It follows that $d w^{\prime}(0)=d w^{\prime \prime}(0)=0$ (since $0=w_{0}=T^{\prime}(0)=\Phi\left(T_{i}, z_{0}\right)$ ). Thus $d w^{\prime \prime \prime}(0)=\Phi_{T} z_{0} T^{\prime \prime}(0)$. If $T^{\prime \prime}(0) \neq 0$ then $w^{\prime \prime \prime}(0) \neq 0$ (since $\Phi_{T}>0$ here). It follows that $w$ assumes negative values in one direction, while $z<1-\delta$. Such solutions leave $N$. Finally, if $T^{\prime \prime}(0)=0$, then $\sigma \tau F_{2 \tau} \tau^{\prime}(0)=0$ (since $w_{0}=T^{\prime}(0)=0$ ). Since $F_{2 \tau} \neq 0$ along $F_{2}=0$, it follows that $\tau^{\prime}(0)=0$. The solution must therefore be the critical point at $X_{i}$.

Lemma 3.8. Suppose that $X_{0} \in \partial N$ does not satisfy any of the conditions in (i) or (ii), and that $T=T_{i}$ and $z_{0} \neq 1-\delta$. Then the solution through $X_{0}$ leaves $N$.

Proof. There are four different cases: (a) $1-\delta<z_{0}<1+\delta$, (b) $z_{i}<z_{0}<1-\delta$, (c) $0<z_{0}<z_{i}$, and (d) $z_{0}=z_{i}$.

(a) In this case, $X_{0}$ is an interior point of $N$.

(b) If $T^{\prime}(0) \neq 0$, then the solution enters the region $\left\{z<1-\delta, T<T_{i}\right\}$ in one direction, and therefore leaves $N$. Suppose then that $T^{\prime}(0)=0$. Since $z_{i}<z_{0}<1-\delta$, it follows that $\tau^{\prime}(0)>0$ (see Figure 2(a)). Moreover, since $X_{0} \in N_{1} \backslash N_{2}$, we have that $w_{0}>0$. Thus

$$
T^{\prime \prime}(0)=\sigma \tau_{0}\left[\partial F_{2} / \partial \tau \tau^{\prime}(0)-q_{0} w_{0}\right]<0,
$$

since $\partial F_{2} / \partial \tau<0$ along $F_{2}=0$. It follows that $T$ has a local maximum at $T_{i}=T(0)$, and the solution leaves $N$ in both directions.

(c) Now suppose that $0<z_{0}<z_{i}$. If $T^{\prime}(0) \neq 0$, the solution leaves $N$; assume then that $T^{\prime}(0)=0$. Since $X_{0} \in N_{1} \backslash N_{2}, w_{0}>0$, so that $z$ must decrease in backward time. By Lemma 3.7, it follows that $z$ decreases along the entire backward half orbit, so that $z$ must converge to a limit in backward time, if the solution is to remain in $N$. It easily follows that $\tau, T$, and $w$ also converge to limits in this direction, and the limiting value of $X$ must be a critical point with $z=0$, i.e., $X_{L^{*}}$ or $X_{L}^{*}$. We now show that this is impossible.

Since $X \in N_{1} \backslash N_{2}$ in backward time, it follows that $T \geqslant T_{i}$ along the entire backward half orbit. Note that $\tau^{\prime}(0)<0$, since $T_{0}=T_{i}, T^{\prime}(0)=0$, and $0<z_{0}<z_{i}$ (see Figure 2(c)). The solution therefore moves into the region $\left\{\tau \geqslant \tau(0), T \geqslant T_{i}\right\}$ in backward time. Since $F_{1}=0$ is independent of $z$ and has negative slope in this region, it follows that $\tau$ cannot have a critical point in backward time, and must therefore remain larger than $\tau(0)$ for all $\xi<0$. However, $\tau_{L}^{*}<\tau_{L^{*}}<\tau(0)$, so that the solution cannot tend to either $X_{L^{*}}$ or $X_{L}^{*}$.

(d) If $z_{0}=z_{i}$, then, as above, it may be assumed that $T^{\prime}(0)=0$. Since $T_{0}=T_{i}$, it follows that $\tau^{\prime}(0)=0$ also. Since $w_{0}>0$, we have that $T^{\prime \prime}(0)=-q_{0} w_{0}<0 . T$ therefore has a local maximum at $T_{i}$, and the solution leaves $N$ in both directions. 
The proof of (iv) will require some additional conditions on $\mu$ and $\lambda$. In particular, let $R$ be the shaded region in Figure 7, and define

(17)

$$
\left\{\begin{array}{c}
a^{\prime}=\max \left\{F_{1}(\tau, T):(\tau, T) \in R\right\}, \\
c^{\prime}=\max \left\{-\partial F_{2} / \partial \tau(\tau, T, z):(\tau, T) \in R, F_{2}(\tau, T, z)=0, \text { and }|1-z| \leqslant \delta\right\} ;
\end{array}\right.
$$

Note that $a^{\prime}$ and $c^{\prime}$ are positive. These constants have already been incorporated in condition (8) (cf. (10)).

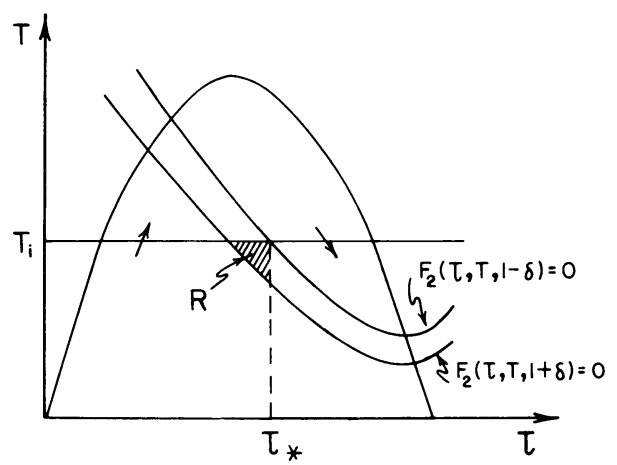

FIGURE 7

Lemma 3.9. Suppose that (8) holds, that $X_{0} \in \partial N$ does not satisfy any of the conditions in (i), and that $z_{0}=1-\delta$. Then for sufficiently small $\varepsilon$ and $\rho=\rho(\varepsilon)$, the solution through $X_{0}$ leaves $N(c f . \S 2 . \mathrm{D})$.

Proof. There are three different cases: (a) $T_{0}<T_{i}$, (b) $T_{0}>T_{i}$, and (c) $T_{0}=T_{i}$.

(a) In this case, $X_{0} \in N_{2} \backslash N_{1}$. If $w_{0} \neq 0$, then the solution enters $\{z<1-\delta, T<$ $\left.T_{i}\right\}$ in one direction, and therefore leaves $N$. If $w_{0}=0$, then $d w^{\prime}(0)=\Phi z<0$ (cf. Figure 1), and the solution leaves $N$ in both directions.

(b) Suppose now that $T_{0}>T_{i}$. If $w_{0}>0$, then $X_{0}$ is an interior point of $N_{1}$. If $w_{0}<0$, then the solution enters $\left\{z<1-\delta, T>T_{i}\right\}$ in forward time, and so, leaves $N$.

Suppose then that $w_{0}=0$. Then $d w^{\prime}(0)=\Phi z_{0}>0$, so that $w<0$ in backward time. Since $\Phi>0$ when $T>T_{i}$, it follows that $z$ cannot have another critical point in backward time while $T>T_{i}$. If $T(\xi)>T_{i}$ for all $\xi>0$, it follows that $z$, and hence $X$, must tend to a limit as $\xi \rightarrow-\infty$. This limit must be a critical point of (6); however, there are no critical points in $\left\{z \geqslant 1-\delta, T \geqslant T_{i}\right\}$. Thus the solution either leaves $N$ or it hits $T=T_{i}$ for some $\xi<0$. Let $\xi_{1}<0$ be the largest such $\xi$. By Lemma 3.6, $z\left(\xi_{1}\right) \leqslant 1$; it also follows that $w\left(\xi_{1}\right) \leqslant 0$ and that $T^{\prime}\left(\xi_{1}\right) \geqslant 0$.

If $z\left(\xi_{1}\right)=1$, then if the solution remains in $N, w\left(\xi_{1}\right)=0$. However, $z=1, w=0$ is an invariant manifold of (6) when $T \leqslant T_{i}$. Since $T^{\prime}\left(\xi_{1}\right) \geqslant 0$, it is easily seen that $T(\xi)<T_{i}$ and $z=1, w=0$, for all $\xi<\xi_{1}$ (cf. Figure 6). Such solutions eventually leave $N$. 
Suppose then that $1-\delta<z\left(\xi_{1}\right)<1$. If $T^{\prime}\left(\xi_{1}\right)=0$ then $(\tau, T) \in R$ (cf. Figure 7); it then follows from (8) and (13) that $T^{\prime \prime}\left(\xi_{1}\right)<0$. This leads to a contradiction, as $T(\xi)>T\left(\xi_{1}\right)$ for $\xi>\xi_{1}$.

It must be that $T^{\prime}\left(\xi_{1}\right)>0$, so that the solution moves into the region $N_{2} \backslash N_{1}$ for $\xi<\xi_{1}$. The claim is that $T^{\prime}(\xi)<0$ for all $\xi<\xi_{1}$. Let $\xi_{2}<\xi_{1}$ be the largest $\xi$ for which $T^{\prime}=0$. Thus $T\left(\xi_{2}\right)<T_{i}$ and $1-\delta<z\left(\xi_{2}\right)<1$. Note that since $T^{\prime}\left(\xi_{1}\right)>0$ and $T\left(\xi_{1}\right)=T_{i}, \tau\left(\xi_{1}\right)<\tau_{*}$, where $\tau_{*}$ is as in Figure 7. Since $\tau^{\prime}>0$ along $T \leqslant T_{i}$, $\tau=\tau^{*}$, it follows that $\tau\left(\xi_{2}\right)<\tau_{*}$ also. Thus $(\tau, T)$ must lie in the region $R$ in Figure 7 at $\xi=\xi_{2}$ (since $F_{2}=0$ at $\xi=\xi_{2}$ ). From (8) and (13), it follows that $T^{\prime \prime}\left(\xi_{2}\right)<0$, which is a contradiction. This proves the claim.

Next note that $w$ must be negative for $\xi<\xi_{1}$. If this were not the case, $z$ would have a local maximum at some point, and would then begin to decrease in backward time. Since $T<T_{i}$ in this direction, and $\Phi<0$ for $T<T_{i}$ and $z<1, z$ would have to decrease as $\xi$ approaches $-\infty$. It follows that $z$ would eventually assume values less than $1-\delta$ while $T<T_{i}$, and so, the solution would leave $N$.

From the above remarks, it follows that $X$ converges to a limit in backward time, where the limiting value of $T$ is less than $T_{i}$, and the limiting value of $\tau$ is less than $\tau_{*}$. Since there are no critical points in this region, the solution must eventually leave $N$.

(c) Suppose that $z_{0}=1-\delta$ and $T_{0}=T_{i}$. We consider the following cases separately: (i) $T^{\prime}(0) w_{0}>0$, or $T^{\prime}(0)>0$ and $w_{0}<0$, (ii) $T^{\prime}(0)$ or $w_{0}=0$, and (iii) $T^{\prime}(0)<0$ and $w_{0}>0$.

(i) Suppose that $T^{\prime}(0) w_{0}>0$; then $T$ and $z$ have the same monotonicity at $\xi=0$, and the solution enters the region $\left\{T<T_{i}, z<1-\delta\right\}$ in one direction. Such solutions leave $N$. If $T^{\prime}(0)>0$ and $w_{0}<0$, the solution enters the region $\left\{T>T_{i}, z\right.$ $<1-\delta, w<0\}$ in forward time, and so, leaves $N$.

(ii) Suppose that $w_{0}=0$ and $T^{\prime}(0) \neq 0$. Since $T_{0}=T_{i}, d w^{\prime}(0)=0$, and since $d^{\prime}\left(T_{i}\right)=0$, it follows that $\left(d w^{\prime}\right)^{\prime}(0)=d w^{\prime \prime}(0)=\Phi_{T} z T^{\prime}(0)$. Since $\Phi_{T}>0$ at $\left(T_{i}, 1-\right.$ $\delta$ ), it follows that $z^{\prime \prime \prime}(0)$ and $T^{\prime}(0)$ have the same sign and that $z$ and $T$ have the same monotonicity for small $|\xi|$. Such solutions leave $N$.

If $T^{\prime}(0)=0$, then $\left(\tau_{0}, T_{0}\right) \in R$ in Figure 7 , since $T_{0}=T_{i}$ and $z_{0}=1-\delta$. Thus by (8) and (13), $T^{\prime \prime}(0)<0$. If $w_{0} \neq 0$, the solution enters $\left\{T<T_{i}, z<1-\delta\right\}$ in one direction, and the solution leaves $N$. If $w_{0}=0$, then $d w^{\prime}(0)=d w^{\prime \prime}(0)=0$, so that $d w^{\prime \prime \prime}(0)=\Phi_{T} z T^{\prime \prime}(0)$. Thus $z^{(4)}(0)$ and $T^{\prime \prime}(0)$ are both negative, and the solution leaves $N$ in both directions.

(iii) Now suppose that $T^{\prime}(0)<0$ and $w_{0}>0$. These points are more difficult to eliminate from $S(N)$. The problem is that the stable manifold of $X_{R}$ is three dimensional (cf. Appendix 3), and it will contain solutions which pass through such boundary points. The idea is to choose $\delta, \varepsilon=\varepsilon(\delta)$, and $\rho=\rho(\varepsilon)$ so small that either $|w|$ exceeds $L$ or $|\pi|$ exceeds $2 K$ at some point along the orbit (cf. (14)). Thus the stable manifold of $X_{R}$ relative to the flow in $S(N)$ must avoid such points.

The first claim is that at time $\bar{\xi}=\delta / 2 L$, there exists a constant $\Lambda>0$ (independent of $w_{0}$ and $\tau_{0}$ ) such that $\Phi(T, z) z<-\Lambda \delta^{2}$ at $\xi=\bar{\xi}$. First note that $T^{\prime}$ is negative on the interval $[0, \bar{\xi}]$; if $T^{\prime}=0$ on this interval, then at the first such point, 
$(\tau, T) \in R$ in Figure 7, so that $T^{\prime \prime}<0$, which leads to a contradiction. Thus $T<T_{i}$ and $z<1$ on $[0, \bar{\xi}]$. In this region, $\Phi(T, z)=-\left(T_{i}-T\right)(1-z)$.

Since $z_{0}=1-\delta$ and $|w| \leqslant L$ in $N$; it follows that if the solution remains in $N$ then $z(\bar{\xi})<1-\delta / 2$. Thus $1-z(\bar{\xi})>\delta / 2$.

The factor $T_{i}-T(\bar{\xi})$ can also be bounded away from zero. Since the constant $\Lambda$ depends on the modifications introduced into the equations and does not appear in the statement of the main theorem, we only sketch the argument. Precise estimates could be given, but the details would not be interesting.

The "worst" possible case is when $\tau_{0}=a$ (see Figure 8), so that $T^{\prime}(0)=0$. We have already seen in (ii) that such points do not lie in $S(N)$. In particular, $T^{\prime \prime}(0)<0$ at this point, and the solution moves into the region $\left\{T<T_{i}, z<1-\delta\right\}$ in backward time (since $w_{0}>0$ ). In particular, $\tau^{\prime}(0)$ is positive and bounded away from zero at this point. It follows that $(\tau, T)$ enters the above region in backward time for all $w_{0} \in[0, L]$ and $\tau_{0} \in[a, b]$, for $b$ sufficiently near $a$ (depending on $\tau^{\prime}(0)=$ $\mu^{-1} F_{1}\left(\tau_{0}, T_{i}\right)$ ). Such solutions do not lie in $S(N)$. For the remaining points $\tau_{0} \geqslant b$ and $w_{0}>0, T^{\prime}(0)$ is bounded away from zero. It follows that $T_{i}-T(\bar{\xi})>\Lambda \delta$ for some $\Lambda>0$ (depending on $b$ and $\lambda^{-1}$ ). Thus $\Phi(T, z) z<-\Lambda \delta^{2}$ for some $\Lambda>0$.

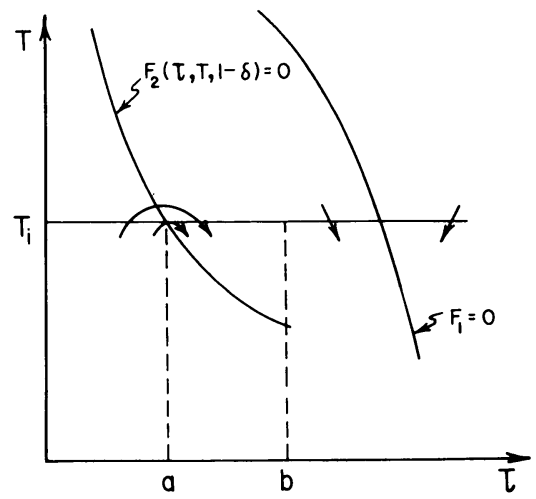

FigURE 8

(We remark that $\Lambda$ depends on $\mu$ and $\lambda$, but it does not depend on $d$. Later, in $\S 5$, we will allow $d$ and $\lambda$ to approach zero. This causes no difficulties with regard to the above estimate, if we perform the homotopy by first letting $d$ approach zero and then allowing $\lambda$ to approach zero. When $d=0$ (after a change of variables), these boundary points can obviously be eliminated from $S(N)$; see $§ 5$.A.)

Now note that $\bar{\xi}=\delta / 2 L$ in the above can be arbitrarily small. It follows that if $\varepsilon<\bar{\xi} / 2$, then $T_{i}-T(\varepsilon)>\Lambda \varepsilon$. Let $\rho=\rho(\varepsilon)=\Lambda \varepsilon$. It follows that $T<T_{i}-\rho$ on $[\varepsilon, \bar{\xi}]$, so that $d(T)=\varepsilon$ on this interval (see $\S 2 . \mathrm{D})$.

It can now be shown that $\pi<-2 K$ at some point along the orbit (see (14)). From the above estimate, we have that $\pi(\bar{\xi})<-\Lambda \delta^{2}$. Moreover, if we regard all other parameters as fixed and let $\varepsilon$ assume small values, $\pi$ satisfies an equation of the form

$$
\pi^{\prime}=-\sigma \varepsilon^{-1} \pi+\vartheta(1),
$$


where the terms $\Theta(1)$ remain bounded as $\varepsilon$ tends to zero (see (15)). It follows that

$$
\begin{aligned}
e^{\sigma} \pi(\varepsilon) & =\pi(\bar{\xi}) \exp \left(\sigma \varepsilon^{-1} \bar{\xi}\right)-\int_{\varepsilon}^{\bar{\xi}} \exp \left(\sigma \varepsilon^{-1} s\right) \mathcal{O}(1) d s \\
& <-\Lambda \delta^{2} \exp \left(\frac{\sigma \delta}{2 \varepsilon L}\right)+\frac{\varepsilon}{\sigma} \vartheta(1)\left[\exp \left(\frac{\sigma \delta}{2 \varepsilon L}\right)+1\right] \\
& <\left[-\Lambda \delta^{2}+\varepsilon \vartheta(1)\right] \exp \left(\frac{\sigma \delta}{2 \varepsilon L}\right)+\varepsilon \mathcal{O}(1) .
\end{aligned}
$$

First choose $\varepsilon$ so small that the coefficient of the exponential is less than $-\Lambda \delta^{2} / 2$. Next, the exponential can be made arbitrarily large by choosing $\varepsilon$ sufficiently small relative to $\delta$. Thus for $\varepsilon$ sufficiently small, $\pi(\varepsilon)<-2 K$. Since $|\pi| \leqslant 2 K$ in $N$, such solutions eventually leave $N$.

This completes the proof of Theorem 3.5. An isolating neighborhood, $N_{s}$, can now be determined. Let $A^{*}, A_{*}$, and $A_{i}$ be small neighborhoods of the critical points at $X_{L}^{*}, X_{L^{*}}$, and $X_{i}$, respectively. Since $X_{i}$ is an attractor for (6) (see Appendix 3), we can take $A_{i}$ to be an attracting neighborhood of $X_{i}$.

The rest point $X_{L^{*}}$ is a saddle with a one-dimensional unstable manifold which is transverse to the plane $z=0$ (see Appendix 3). Denote the orbit in this manifold along which $z>0$ near $X_{L^{*}}$ by $\Re^{u}$. It will be shown later that $\Re^{u} \subset S\left(N_{w}\right)$ (see §4.C), so that $z<\bar{z}$ along this orbit.

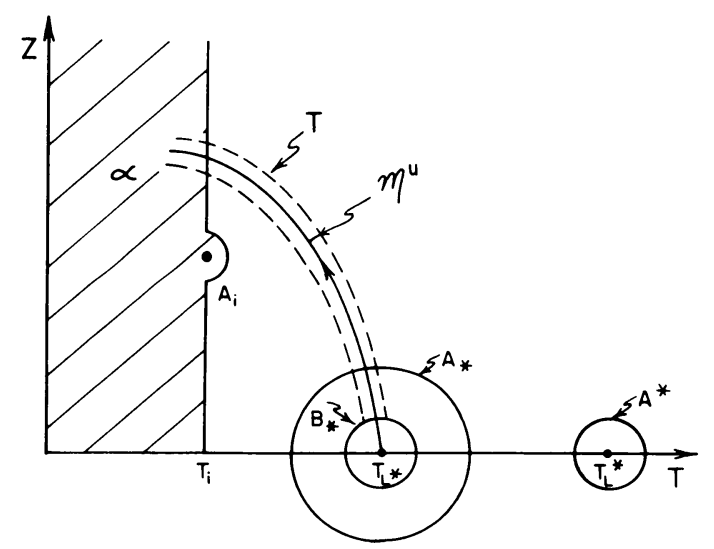

FIGURE 9

Now let $\alpha=\left\{X: T \leqslant T_{i}, z \leqslant \bar{z}\right\} \cup A_{i}$. The projection on the $(T, z)$ plane is depicted in Figure 9.

LEMMA 3.10. The $z$ component along $\Re^{u}$ is monotone increasing up until the first time $\mathfrak{T}^{u}$ hits $\partial \alpha$. This orbit hits $\partial \alpha$ for some $\xi=\xi_{1}<+\infty$, and for $\xi>\xi_{1}$, the orbit enters the interior of $\alpha$. In particular, if $T=T_{i}$ at $\xi_{1}$, then $T^{\prime}\left(\xi_{1}\right)<0, z\left(\xi_{1}\right) \in\left[z_{i}, \bar{z}\right)$, and $w\left(\xi_{1}\right)>0$.

Proof. If $T>T_{i}$, then $\Phi z>0$ so that $z$ must have a local minimum at any point with $z^{\prime}=0, T>T_{i}$. Since $z^{\prime}>0$ along $\Re^{u}$ near $X_{L^{*}}$, it follows that $z$ is monotone increasing until $\mathfrak{T}^{u}$ hits $\partial \alpha$. 
If $\Re^{u} \cap \partial \alpha=\varnothing, z$ must increase along the entire orbit. Since $\Re^{u} \subset S\left(N_{w}\right), z$ (and hence, $\tau, T$, and $w$ ) must tend to limits in forward time. However, there are no critical points in the region $z>0$ and $X \notin \alpha$, so that $\Re \mathcal{R}^{u}$ must hit $\partial \alpha$ at some point.

If $X\left(\xi_{1}\right) \in \partial A_{i}$, then the solution immediately enters the interior of $A_{i}$ in forward time, since $A_{i}$ is an attracting neighborhood of $X_{i}$. If $T\left(\xi_{1}\right)=T_{i}$, then we must have that $T^{\prime}\left(\xi_{1}\right) \leqslant 0$. If $z\left(\xi_{1}\right) \geqslant z_{i}$, then the arguments in (b) and (d) of Lemma 3.8 show that $T^{\prime \prime}\left(\xi_{1}\right)<0$ if $T^{\prime}\left(\xi_{1}\right)=0$, which is impossible; thus $T^{\prime}\left(\xi_{1}\right)<0$ in this case. If $z\left(\xi_{1}\right)<z_{i}$, an argument similar to that of (c) in Lemma 3.8 shows that the solution must leave $N$ in backward time, which is also impossible. Thus $z\left(\xi_{1}\right) \geqslant z_{i}$ and $T^{\prime}\left(\xi_{1}\right)<0$. If $w\left(\xi_{1}\right)=0$, then since $T\left(\xi_{1}\right)=T_{i}, w^{\prime}\left(\xi_{1}\right)=0$, we have that $d w^{\prime \prime}\left(\xi_{1}\right)=$ $\Phi_{T} z T^{\prime}(0)<0$. Thus $z^{\prime \prime \prime}\left(\xi_{1}\right)<0$, which leads to a contradiction, since $z$ is increasing for $\xi<\xi_{1}$.

Now let $B_{*} \subset A_{*}$ be a (much) smalter neighborhood of $X_{L^{*}}$. Let $T$ be a narrow tubular neighborhood of the solution segment along $\Re^{u}$ outside of $B_{*}$ extending beyond the point at $\xi=\xi_{1}$ where $\mathfrak{T}^{u}$ enters $\alpha$.

The final neighborhood, $N_{s}$, is defined to be

$$
N_{s}=\left(N \cup A^{*}\right) \backslash\left(A_{i} \cup T \cup B_{*}\right) .
$$

THEOREM 3.11. $N_{s}$ is an isolating neighborhood.

Proof. Since $X_{i}, X_{L^{*}} \notin N_{s}$ and $X_{L}^{*}$ is an interior point of $N_{s}$, these critical points do not lie in $\partial N_{s}$. Also, the orbit along which $z=w=0$ that connects $X_{L}^{*}$ to $X_{L^{*}}$ enters the neighborhood $B_{*}$ and is therefore not in $S\left(N_{s}\right)$. Thus by Theorem 3.5, it need only be shown that $S\left(N_{s}\right)$ is disjoint from the new boundary points, i.e., $\left[\partial N \cap \partial\left(A_{i} \cup B_{*} \cup T\right)\right] \cup \partial A^{*}$.

The argument for points in $\partial A^{*}$ is exactly the same as for points in $\partial A_{r}$ in Lemma 3.2 , and will be omitted.

If $X_{0} \in \partial A_{i}$, then the solution through $X_{0}$ enters the interior of $A_{i}$ in positive time, since $A_{i}$ is an attracting neighborhood. The solution therefore leaves $N_{s}$.

Suppose that $X_{0} \in \partial T$. If $T$ is thin enough, the solution through $X_{0}$ will closely approximate $\mathfrak{T}^{u}$ on finite time intervals. By Lemma 3.11, $\mathfrak{T}^{u}$ enters the interior of the region $\alpha$ in finite time, and so, leaves $N_{s}$, since $z<\bar{z}<1-\delta$ along $\Re^{u}$. It follows that the solution through $X_{0}$ leaves $N_{s}$ in forward time.

Finally, suppose that $X_{0} \in \partial B_{*}$. Since $X_{L^{*}}$ is a saddle with a one-dimensional unstable manifold, the solution through $X_{0}$ either tends to $X_{L^{*}}$ in positive time (and so, leaves $N_{s}$ ), or it eventually exits the neighborhood $A_{*} \supset B_{*}$ in positive time. Moreover, it follows from the Hartman linearization theorem that when the solution through $X_{0}$ leaves $A_{*}$ in forward time, it must be close to the unstable manifold of $X_{L^{*}}$ provided that $B_{*}$ is sufficiently smaller than $A_{*}$. It follows that the solution either enters the region $\{z<0\}$ (and so, leaves $N_{s}$ ), or it hits a point in $T \cap \partial A_{*}$ (provided that $B_{*}$ is sufficiently small). In the latter case, the remarks in the preceding paragraph show that the solution eventually enters $\alpha$, and so, leaves $N_{s}$. 
4. The index of $S\left(N_{w}\right)$. It would be difficult to compute the index $h\left(S\left(N_{w}\right)\right)$ directly from the definition of $h$ in Appendix 4. This is avoided by exploiting the homotopy invariance of $h$. In particular, $h$ is constant under homotopies of both the flow, (6), and of $N_{w}$, such that $S\left(N_{w}\right)$ is interior to $N_{w}$ throughout the deformation. Finally, it is shown that $S\left(N_{w}\right)$ (with respect to (6)) contains the portion $\Re^{u}$ of the unstable manifold of $X_{L^{*}}$.

A. Continuation to Majda's model. The homotopy consists of allowing $d$, and then $\lambda$ to approach zero. (We first deform $d$ by decreasing $D$ until $d \equiv \varepsilon$; we then let $\varepsilon$ approach zero.) It is essential that the homotopy be taken in this order, since the estimates of Lemma 3.4 require that $\varepsilon$ be chosen small relative to $\lambda$.

At the limiting values of the homotopy parameters, the equations are singular. We shall therefore need to introduce certain changes of variables in order to obtain a better description of the behavior of solutions when $d$ and $\lambda$ are near zero.

Suppose that $D$ has been decreased to $\varepsilon$, so that $d(T)=\varepsilon$ for all $T$. Let $\pi$ be as in (14); it will be convenient to introduce a new variable $p(\xi)$ defined by (see (15))

$$
p^{\prime}=\left(-\frac{\sigma}{\varepsilon} p+\vartheta(1)\right) \beta,
$$

where $\beta \in[\varepsilon, 1]$. Thus when $\beta=1, p=\pi$. We now consider the system

$$
\begin{cases}\mu \tau^{\prime}=F_{1}, & z^{\prime}=\sigma^{-1} \Phi(T, z) z-\sigma^{-1} p, \\ \lambda T^{\prime}=\sigma \tau F_{2}, & p^{\prime}=\left(-\frac{\sigma}{\varepsilon}+\vartheta(1)\right) \beta,\end{cases}
$$

where the $\theta(1)$ terms remain bounded as $\varepsilon$ tends to zero.

We shall also need a suitable isolating neighborhood for (19). To this end, let $l$ be a positive constant, and let $I_{p}=\{p:|p| \leqslant l\}$. Let $\hat{A}_{r}$ be the projection of $A_{r}$ on the $(\tau, T, z)$ plane, and define

$$
R_{w}=(B \times\{z: 0 \leqslant z \leqslant \bar{z}\}) \cup \hat{A}_{r} .
$$

Finally, let $M_{w}=R_{w} \times I_{p}$.

LEMMA 4.1. (a) Let $\beta=1$; for sufficiently small $\varepsilon, M_{w}$ is an isolating neighborhood for $(19)_{1}$, and the index of $S\left(M_{w}\right)$ with respect to $(19)_{1}$ equals that of $S\left(N_{w}\right)$ with respect to (6).

(b) With $\varepsilon$ as in (a), the index of $S\left(M_{w}\right)$ with respect to $(19)_{\beta}$ is the same for all $\beta \in[\varepsilon, 1]$.

Proof. (a) Let $\tilde{N}_{w}$ be the image of $N_{w}$ in the $(\tau, T, z, p)$ plane under the change of variables $w \rightarrow \pi(=p)$. It is easily checked that this is $a 1: 1$ mapping of $N_{w}$ onto its image. It follows that $\tilde{N}_{w}$ is an isolating neighborhood for $(19)_{1}$ and that the index of $S\left(N_{w}\right)$ with respect to (6) equals that of $S\left(\tilde{N}_{w}\right)$ with respect to (19) .

The projection of $\tilde{N}_{w}$ on the $p$-axis for each $(\tau, T, z)$ contains a uniform interval $I_{p}$ about $p=0$, with the exception of certain points in the image of $A_{r}$ with $z<0$. However, it is easily checked that $A_{r}$ need only be chosen so that $(\tau, T, z)$ is near ( $\tau_{L^{*}}, T_{L^{*}} 0$ ) when $X \in A_{r}$ (cf. Lemma 3.2). Thus $A_{r}$ can be increased so that its projection on the $w$-axis contains a uniform interval $|w| \leqslant L$. Since this does not 
affect the maximal invariant set in $N_{w}$, the index remains the same as $A_{r}$ is increased in this manner. It now follows that $\tilde{N}_{w}$ contains a uniform interval $I_{p}$ for some $l>0$ and for each $(\tau, T, z) \in R_{w}$.

We now claim that $S\left(\tilde{N}_{w}\right)=S\left(M_{w}\right)$ for sufficiently small $\varepsilon$ and $\beta=1$. This follows from the $p^{\prime}$ equation in (19). In particular, $|p| \leqslant R \varepsilon$ where $R>\sigma^{-1} \Theta(1)$, along solutions in $S\left(\tilde{N}_{w}\right)$ and $S\left(M_{w}\right)$. For example, if $p=\varepsilon R$ then $p^{\prime}<0$ at this point, so that $p$ increases without bound in backward time. If $p=-R \varepsilon$ the argument is similar. Now choose $\varepsilon$ so small that $\varepsilon R<l$. By $§ 3$.A, orbits in $S\left(\tilde{N}_{w}\right)$ and $S\left(M_{w}\right)$ are disjoint from $\partial R_{w} \times I_{p}$, and by the above, they are also disjoint from $R_{w} \times \partial I_{p}$. Thus these invariant sets are the same.

(b) The argument of the preceding paragraph shows that $|p| \leqslant R \varepsilon$ along solutions in $S\left(M_{w}\right)$. Since this argument is independent of $\beta, S\left(M_{w}\right)$ is an isolated invariant set for $(19)_{\beta}$ for each $\beta \in[\varepsilon, 1]$.

Now let $\beta=\varepsilon$ and let $\varepsilon$ tend to zero. From Lemma 4.1 it follows that the index of $S\left(N_{w}\right)$ with respect to (6) equals that of $S\left(R_{w} \times I_{p}\right)$ with respect to

$$
\begin{cases}\mu \tau^{\prime}=F_{1}(\tau, T), & z^{\prime}=\sigma^{-1} \Phi(T, z) z, \\ \lambda T^{\prime}=F_{2}(\tau, T, z), & p^{\prime}=-\sigma p .\end{cases}
$$

Note that $p=0$ is an invariant manifold of (20) and that $R_{w}$ is an isolating neighborhood for the $(\tau, T, z)$ flow in this manifold.

We next let $\lambda$ tend to zero. To this end, define a new variable $q$ by

$$
q(\xi)=\lambda T^{\prime}(\xi)=\sigma \tau F_{2}(\tau, T, z) .
$$

Since $F_{2} / \partial T=-c<0, q$ measures how far away the solution is from $F_{2}=0$.

The surface $F_{2}=0$ defines $T$ as a function of $(\tau, z)$; this function will be denoted by $T_{0}(\tau, z)$. Let $f(\tau, z)=F_{1}\left(\tau, T_{0}(\tau, z)\right)$ and $g(\tau, z)=\sigma^{-1} \Phi\left(T_{0}(\tau, z), z\right) z$. From (21) it follows that $\left|f-F_{1}\right|=\theta(1) q$ and that $\left|\sigma^{-1} \Phi z-g\right|=\theta(1) q$ as $q$ tends to zero.

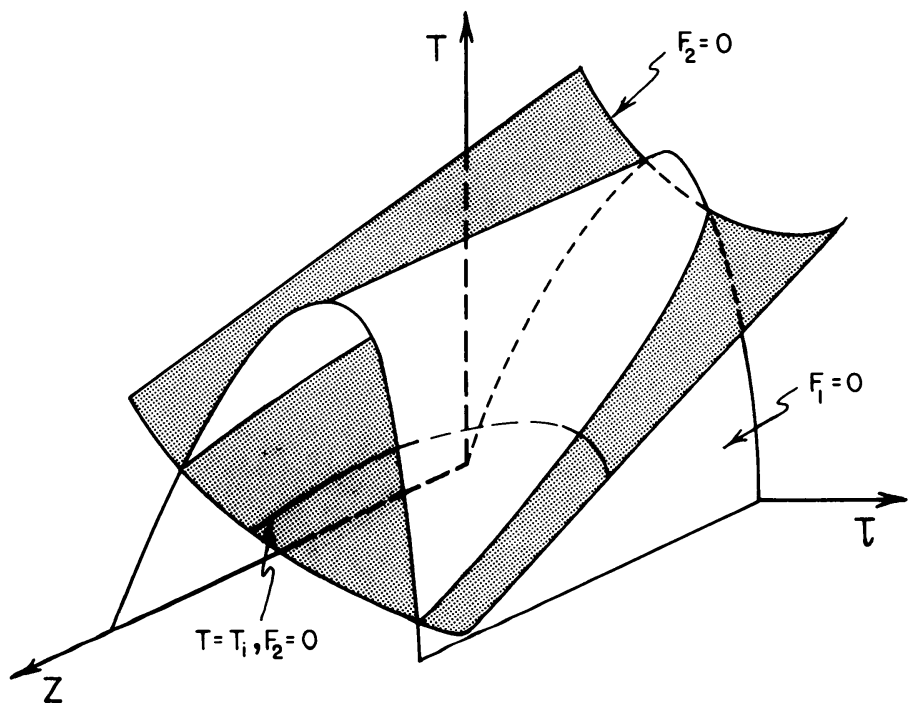

FIGURE 10 
Let $H_{w}$ be the projection of $R_{w}$ on the $\tau-z$ plane (see Figure 11), and let $\tilde{R}_{w}$ be the image of $R_{w}$ under the mapping $(\tau, T, z) \rightarrow(\tau, z, q)$. As before, it follows that the projection of $R_{w}$ on the $q$-axis contains a uniform interval $I_{q}=\{q:|q| \leqslant l\}$ about $q=0$, provided that $\hat{A}_{r}$ is modified in a suitable manner when $z<0$.

The equations (20) in the variables $(\tau, z, p, q)$ take the form

$$
\begin{cases}\mu \tau^{\prime}=f(\tau, z)+\vartheta(1) q, & p^{\prime}=-\sigma p, \\ z^{\prime}=g(\tau, z)+\vartheta(1) q, & q^{\prime}=\left[\frac{-\sigma \tau c}{\lambda} q+\vartheta(1)\right] \beta,\end{cases}
$$

when $\beta=1$.

LEMMA 4.2. For sufficiently small $\lambda>0, H_{w} \times I_{p} \times I_{q}$ is an isolating neighborhood for $(22)_{\beta}$ for each $\beta \in[\lambda, 1]$. When $\beta=1$, it isolates the same invariant set as $\tilde{R}_{w} \times I_{p}$.

The proof is the same as that of Lemma 4.1 and will be omitted.

From Lemma 4.2, it follows that the index of $S\left(N_{w}\right)$ with respect to (6) equals that of $S\left(H_{w} \times I_{p} \times I_{q}\right)$ with respect to $(22)_{\lambda}$, and that $|q|=\vartheta(1) \lambda$ along bounded solutions. Now let $\lambda$ tend to zero to obtain

$$
\begin{array}{ll}
\mu \tau^{\prime}=f(\tau, z)+\vartheta(1) q, & p^{\prime}=-\sigma p, \\
z^{\prime}=g(\tau, z)+\vartheta(1) q, & q^{\prime}=-\sigma c \tau q .
\end{array}
$$

Since $q \equiv 0$ along bounded solutions of this system, the equations can be deformed to

$$
\begin{array}{ll}
\mu \tau^{\prime}=f(\tau, z), & p^{\prime}=-\sigma p, \\
z^{\prime}=g(\tau, z), & q^{\prime}=-\sigma q,
\end{array}
$$

without affecting the maximal invariant set (and hence, the index). Thus the $(p, q)$ equations decouple from the $(\tau, z)$ equations. It follows that the index $h\left(S\left(N_{w}\right)\right)$ equals $h\left(S\left(H_{w}\right)\right)$, since the $(p, q)$ equations are linear with an attracting critical point at the origin (see Conley [1]). It therefore suffices to compute $h\left(S\left(H_{w}\right)\right)$ with respect to

$$
\left\{\begin{array}{l}
\mu \tau^{\prime}=f(\tau, z) \\
z^{\prime}=g(\tau, z)
\end{array}\right.
$$

B. Computation of the index. It can be seen that $f=0$ is as depicted in Figure 11 . Also, $g=0$ consists of $z=0$, a portion of the line $z=1$, and a third curve which corresponds to the trace of $F_{2}=0$ in the plane $T=T_{i}$ (see Figure 10). The equations (23) are similar to Majda's model [6]. (Majda's "fluid" variable corresponds to density, whereas the fluid variable used here is specific volume. Thus our equations more closely resemble Majda's after the change of variables $\rho=\tau^{-1}$.) The index can now be computed. 


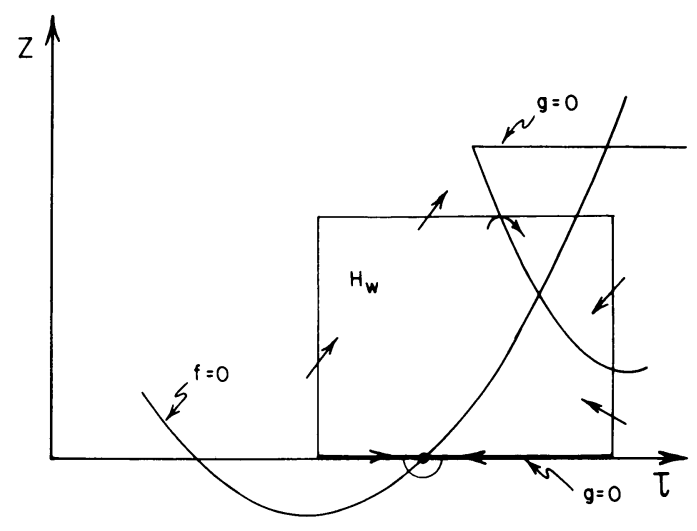

FIGURE 11

LEMMA 4.3. $h\left(S\left(H_{w}\right)\right)=\overline{0}$, i.e., the homotopy type of a pointed point.

Proof. It follows from previous remarks that $H_{w}$ is an isolating neighborhood for (23). We compute the index by deforming $g=0$ in such a manner that the two critical points in $H_{w}$ disappear at the end of the homotopy. Note that the only point where $S\left(H_{w}\right)$ can intersect $\partial H_{w}$ is the point in $g=0$ with $z=\bar{z}$ (see Figure 11). The orbit through this point can be forced to leave $H_{w}$ if $\mu$ is small enough. Thus we may have to decrease $\mu$ as $g=0$ is deformed in order to ensure that $H_{w}$ is an isolating neighborhood throughout the homotopy.

The homotopy begins by deforming the curved portion of $g=0$ to a vertical line (see Figure 12(a)). This line then meets $z=0$ at some point to the right of $\tau=\tau_{L^{*}}$. These two branches are then "pulled apart" so that $g=0$ consists of two components (see Figure 12(b)).

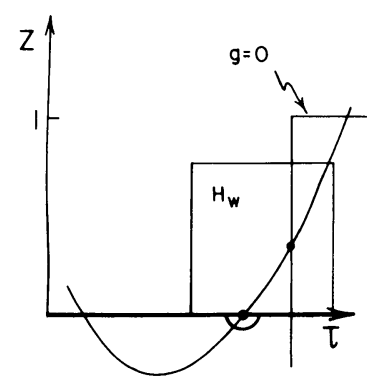

(a)

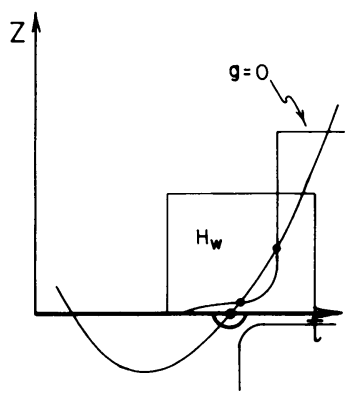

(b)

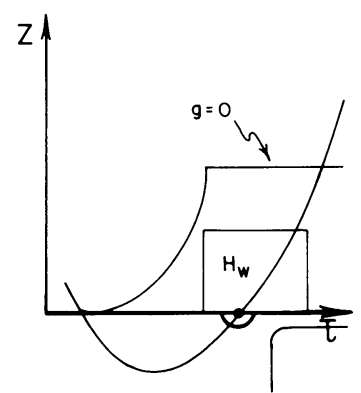

(c)

FigURE 12

As the component in $z>0$ is raised upwards and to the left, the two critical points in $H_{w}$ move together, coalesce, and finally disappear. At the end of the homotopy, $g<0$ everywhere in $H_{w}$. It follows that every solution which hits a point in $H_{w}$ eventually leaves $H_{w}$, so that $S\left(H_{w}\right)=\varnothing$. It follows that $h\left(S\left(H_{w}\right)\right)=\overline{0}$ (see Appendix 4). 
It follows from the above that $h\left(S\left(N_{w}\right)\right)$ with respect to the original equations (6) equals $\overline{0}$. Note that $N_{w}$ contains two hyperbolic critical points $X_{i}$ and $X_{L^{*}}$. The index of a hyperbolic critical point with $k$ eigenvalues with positive real part is $\Sigma^{k}$, i.e., a pointed $k$-sphere. If $S\left(N_{w}\right)$ were equal to the two critical points $\left\{X_{i}, X_{L^{*}}\right\}$, it would follow that $h\left(S\left(N_{w}\right)\right)=h\left(X_{i}\right) \vee h\left(X_{L^{*}}\right)=\Sigma^{0} \vee \Sigma^{1}$, since $X_{i}$ is an attractor and $X_{L^{*}}$ has one positive eigenvalue (see Appendix 4). Since this is not the case, it follows that $S\left(N_{w}\right)$ contains nonconstant solutions. Moreover, by the sum formula in Appendix 4, it follows that $X_{i}$ cannot be an isolated point in $S\left(N_{w}\right)$ (otherwise $S\left(N_{w}\right)$ would have nonzero index). In the case of the limiting equations (23), it is clear that $\left(\tau_{L^{*}}, 0\right)$ is a repellor relative to $S\left(H_{w}\right)$, i.e., $S\left(H_{w}\right)$ contains the portion of the unstable manifold of $\left(\tau_{L^{*}}, 0\right)$ along which $z>0$. In the following section, we show that this is also true for the original equations.

C. The global behavior of $\Re^{u}$. We now show that $\Re^{u} \subset S\left(N_{w}\right)$. To this end, some additional conditions on $\lambda, \mu$, and $D$ will have to be prescribed.

Let $z \in\left(0, z_{i}\right)$ be given, and define $F_{2}(\tau, T)=F_{2}\left(\tau, T_{1} z\right)$. Let $T$ be the value of $T$ where $\underline{F}_{2}=0$ intersects $F_{1}=0$, and let $T_{0} \in\left(T_{i}, \underline{T}\right)$. Finally, let $l$ be the indicated line in Figure 13, and let $S_{L}\left(S_{R}\right)$ be the region below $T=T_{0}$ and to the left (right) of $l$. Positive constants $a^{\prime \prime}$ and $b^{\prime \prime}$ can be defined which bound $-F_{1}$ away from zero in $S_{R}$ and $\sigma \tau \underline{F}_{2}$ away from zero in $S_{L}$, respectively. These constants have already been incorporated into the definition of $a$ and $b$ in (10).

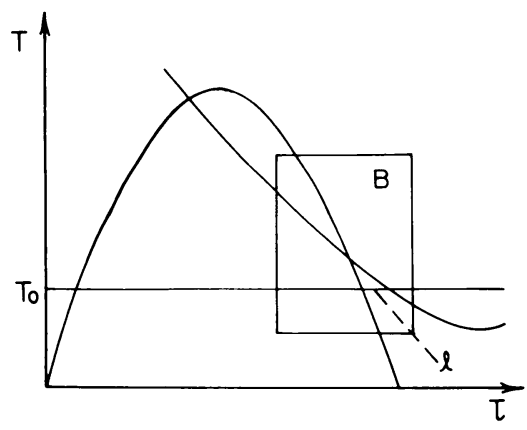

FigURE 13

Lemma 4.4. Suppose that (7), (8), and (9) hold. Then $X_{L^{*}}$ is a repellor relative to $S\left(N_{w}\right)$.

The details of the argument closely parallel those of Lemmas 3.3 and 3.4. We will therefore only sketch the proof.

Suppose that an orbit in $S\left(N_{w}\right)$ tends to $X_{L^{*}}$ in positive time. Since $\Phi>0$ when $T>T_{i}$, it follows that $z$ must be monotone decreasing along the orbit extending from the largest time that $T=T_{i}$ until $\xi=+\infty$. If the orbit remains in $N_{w}$ for all time, it follows that $T=T_{0}$ at some point. Assume that this occurs at $\xi=0$, and that $T(\xi)>T_{0}$ for all $\xi>0$. 
The first case is if $0 \leqslant z(0) \leqslant \underline{z}$. Arguments similar to those of Lemma 3.3 show that the $(\tau, T)$ components eventually leave $B$ in backward time, under suitable conditions on $\mu$ and $\lambda$.

If $\underline{z} \leqslant z \leqslant \bar{z}$ an argument similar to Lemma 3.4 applies. In particular, we introduce the variable $\pi=D w^{\prime}$ (note that $d=D$ for $T \geqslant T_{i}$ ). Since $\phi=\Phi>0$ for $T>T_{i}$ and $z^{\prime}(0)<0$, it follows that $\pi(0) \geqslant \phi\left(T_{0}\right) z$. Moreover, $\pi^{\prime}$ satisfies an equation of the form

$$
\pi^{\prime}=\left(-\sigma D^{-1}+\vartheta(1)\right) \pi+\theta(1) \lambda^{-1} .
$$

Let $\xi_{1}=-\left(T_{0}-T_{i}\right) \lambda /(2 M)$. Then $T \geqslant T_{i}$ for $\xi \in\left[\xi_{1}, 0\right]$. If $P$ is as in (16) and $Q=\pi(0)$, then $\pi\left(\xi_{1}\right)>2 K$ provided that $D$ satisfies a condition analogous to the condition on $\varepsilon$ in (16). Since the denominator on the right-hand side of this inequality contains a factor of $\lambda^{-1}$, the cendition is implied by (9), where

$$
\Gamma=\sigma Q /\left[\lambda\left(P+Q P+(2 K+Q)\left|\xi_{1}\right|^{-1}\right)\right] .
$$

(Since $P$ and $\left|\xi_{1}\right|^{-1}$ each contain a factor of $\lambda^{-1}, \Gamma$ is bounded as $\lambda$ tends to zero.)

From Lemma 4.4 and the remarks at the end of $\S 4 . \mathrm{B}$, it follows that $\Re^{u} \subset S\left(N_{w}\right)$.

5. The index of $S\left(N_{s}\right)$. In this section, we continue the flow of (6) in $N_{s}$ to the product system (22) with $\lambda=0$, via the changes of variable $w \rightarrow p$ and $T \rightarrow q$. The methods and notation are similar to those used in $\S 4$.

A. Continuation to Majda's equation. The homotopy is the same as that of §4.A. Thus we continue (6) through the sequence of systems (19), (20), and (22), as $d$, and then $\lambda$ approach zero. Regions $R_{s}$ and $H_{s}$ analagous to $R_{w}$ and $H_{w}$ are defined in the obvious manner. (Note that neighborhoods of $X_{L^{*}}, X_{i}$, and the unstable manifold of $X_{L^{*}}$ will need to be excised from these lower dimensional regions.)

The difficulty is that the image of $\tilde{N}_{s}$ in the $(\tau, T, z, p)$ plane will not always project onto an interval $I_{p}$ on the $p$-axis containing zero in its interior. For example, if $T=T_{i}$ and $z<1-\delta$, then the fiber over $(\tau, T, z)$ in $\tilde{N}_{s}$ is a half interval $\{-\sigma L \leqslant p \leqslant 0\}$, since $0 \leqslant w \leqslant L$ at all such points.

This is remedied by increasing $N_{s}$ through a family of isolating neighborhoods, $N_{s}(l)=N_{s} \cup N_{1}(l)$, where

$$
N_{1}(l)=C_{1} \times\{(z, w): 0 \leqslant z \leqslant 1-\delta,-l \leqslant w \leqslant L\} .
$$

LEMMA 5.1. $N_{s}(l)$ is an isolating neighborhood for (6) for all $l \geqslant 0$.

Proof. Since isolating neighborhoods persist under perturbation $N_{s}(l)$ is isolating for all sufficiently small $l \geqslant 0$.

Let $l_{0}$ be the smallest positive $l$ for which $N_{s}(l)$ is not isolating. Since $S\left(N_{s}\right)=$ $S\left(N_{s}(l)\right)$ for all $l<l_{0}$, it follows that some solution in $S\left(N_{s}\left(l_{0}\right)\right)$ must pass through a "new" boundary point, i.e., a point $X_{0}$ with $T_{0} \geqslant T_{i}, z_{0} \leqslant 1-\delta$, and $w_{0}=-l_{0}$. Since $\Phi z_{0} \geqslant 0$ when $T_{0} \geqslant T_{i}$, we have that $d w^{\prime}=\sigma l_{0}+\Phi z_{0}>0$. If $z_{0}<1-\delta$, then $w<-l_{0}$ in backward time, and the solution leaves $N_{s}\left(l_{0}\right)$. If $z_{0}=1-\delta$, then the solution enters $N_{2}$ in backward time while $w<0$. The argument in (b) of Lemma 3.9 shows that the solution leaves $N_{s}\left(l_{0}\right)$ in backward time. 
From Lemma 5.1, it follows that $h\left(S\left(N_{s}\right)\right)=h\left(S\left(N_{s}(L)\right)\right)$. Now let $\tilde{N}_{s}(L)$ be the image of $N_{s}(L)$ in the $(\tau, T, z, p)$ plane. It follows that the projection of $\tilde{N}_{s}(L)$ on the $p$-axis contains a uniform interval $I_{p}=\{p:|p| \leqslant l\}$, for some $l>0$. As in $\S 4$, it follows that $h\left(S\left(N_{s}\right)\right)$ with respect to (6) equals $h\left(S\left(R_{s} \times I_{p}\right)\right)$ with respect to (20).

A similar problem is encountered as $\lambda$ tends to zero. In particular the projection on the $q$-axis of the image of $H_{s} \times I_{p}$ in the $(\tau, z, p, q)$ plane will not contain the interval $I_{q}$ when $T$ is equal to or slightly larger than $T_{i}$ and $z<1-\delta$. However, since $R_{s} \times I_{p}$ is an isolating neighborhood, $S\left(R_{s} \times I_{p}\right)$ is disjoint from such points; for small $\lambda, S\left(R_{s} \times I_{p}\right)$ will lie within $\Theta(\lambda)$ of $F_{2}=0$. Thus if $R_{s} \times I_{p}$ is increased by including a small neighborhood of such points, this will not affect the maximal invariant set and the index will remain the same. Moreover, the projection on the $q$-axis of the image of the (modified) region $R_{s} \times I_{p}$ in the $(\tau, z, p, q)$ plane will contain a uniform interval $I_{q}$ for sufficiently small $\lambda$. Thus the flow can be continued to that of (22) with $\lambda=0$ in the region $H_{s} \times I_{p} \times I_{q}$ (see Figure 14).

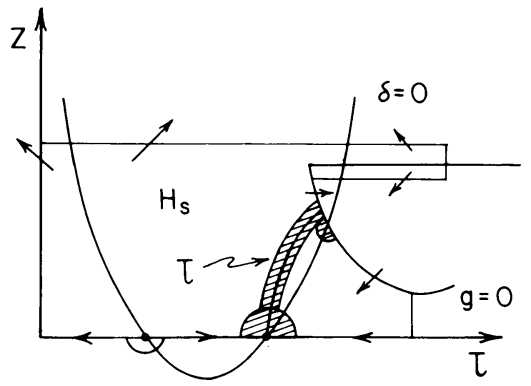

FIGURE 14

B. The index of $S\left(N_{s}\right)$. From the remarks in A, it follows that $h\left(S\left(N_{s}\right)\right)$ equals $h\left(S\left(H_{s}\right)\right)$ with respect to (23). $H_{s}$ is the indicated region in Figure 14. Note that neighborhoods of two of the critical points have been excised, together with a neighborhood $t$ of the unstable manifold of $\left(\tau_{L^{*}}, 0\right)$.

LEMMA 5.2. The index $h\left(S\left(H_{s}\right)\right)=\overline{0}$.

Proof. Note that every orbit which hits a point in $H_{s}$ to the right of $t$ and below the curve $g=0, T=T_{i}$, eventually enters the region $T \geqslant T_{i}$ while $z<1-\delta$, and so, leaves $H_{s}$. Let $D$ be this set of points. It follows that $h\left(S\left(H_{s}\right)\right)=h\left(S\left(H_{s} \backslash D\right)\right)$.

The homotopy begins by deforming $g=0$ inside $D$ in the manner described in §4.B. In particular, $g=0$ is pulled apart into two components, and the critical points corresponding to $X_{L^{*}}$ and $X_{i}$ are cancelled. This is indicated in Figure 15(b). Next, the "upper" component of $g=0$ is deformed to a vertical line through $z=1$. Finally, $f=0$ is translated upwards. The two critical points move together, cancel, and finally disappear, as indicated in Figure 15(d). Here, $f>0$ in $H_{s}$, and every solution leaves $H_{s}$ in finite time. Thus $S\left(H_{s}\right)=\varnothing$ and $h\left(S\left(H_{s}\right)\right)=\overline{0}$.

Since $h\left(X_{R}\right)=\Sigma^{1}$ and $h\left(X_{L}^{*}\right)=\Sigma^{2}$ it follows that $S\left(N_{s}\right) \supsetneqq\left\{X_{L}^{*}, X_{R}\right\}$ (cf. $\S 4$.B and Appendix 3), and that $X_{L}^{*}$ is not isolated in $S\left(N_{s}\right)$. Since $z>0$ and $z^{\prime}>0$ along nonconstant solutions in $S\left(N_{s}\right)$ near $X_{L}^{*}$, it follows that the nonconstant solution in $S\left(N_{s}\right)$ lies in the unstable manifold of $X_{L}^{*}$. 


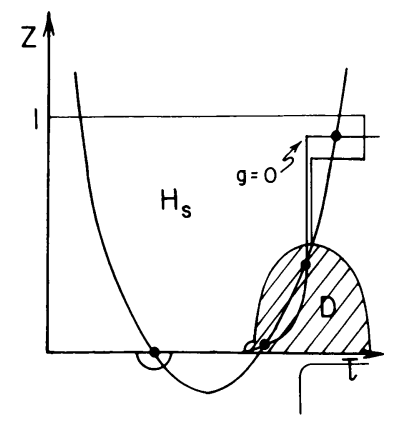

(a)

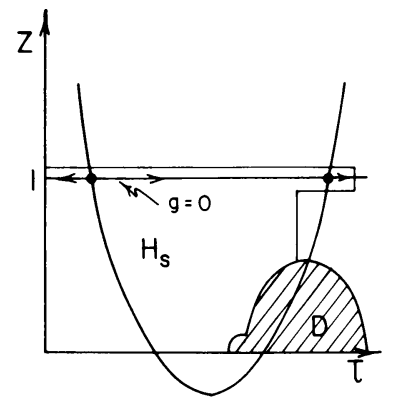

(c)

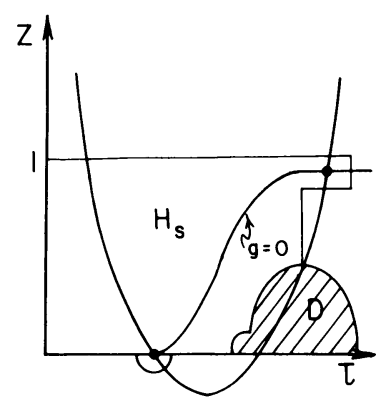

(b)

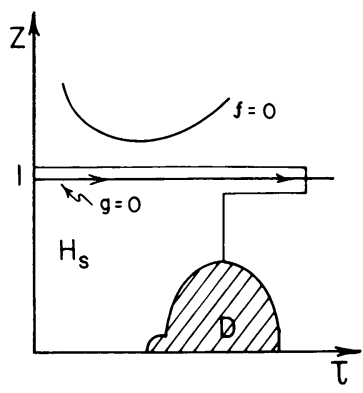

(d)

FIGURE 15

With a little effort, it could be shown that this orbit is a connection from $X_{L}^{*}$ to $X_{R}$. However, we are only interested in the behavior of solutions of (6) in the unmodified region, $T \geqslant T_{i}$. The important information about $S\left(N_{s}\right)$ is therefore contained in the preceding paragraph.

6. Proof of Theorem 2.1. As mentioned in the introduction, we use the behavior of the solutions obtained in $\S \S 4$ and 5 of the modified equations, (6), to construct certain solutions $X_{0}(\xi)$ and $X_{1}(\xi)$ of the original flow, $(6)_{0}$, which lie in the unstable manifold of $X_{L}^{*}$ and which tend to critical points $C\left(z_{0}\right)$ and $C\left(z_{1}\right)$ along the curve $C(z)$ at $+\infty$, where $z_{0}<1<z_{1}$. A shooting argument is then invoked along a portion of the unstable manifold of $X_{L}^{*}$ to obtain the desired solution, $X_{s}(\xi)$.

A. The orbits $X_{0}(\xi)$ and $X_{w}(\xi)$. Let $\Re_{*}$ denote the portion of the unstable manifold of $X_{L^{*}}$ with respect to $(6)_{0}$ along which $z>0$. Thus $\Re_{*}$ coincides with $\Re^{u}$ extending from $X_{L^{*}}$ until the first point $X_{0}$ along $\Re^{u}$ where $T=T_{i}$. The following lemma shows that $\Re_{*}$ is the solution curve of $(6)_{0}$ corresponding to $X_{w}(\xi)$ of Theorem 2.1.

LEMMA 6.1. Suppose that a solution of (6) $)_{0}$ hits a point $X_{0}$ at $\xi=0$ such that $T_{0}=T_{i}$, $T^{\prime}(0)<0, w_{0}>0$, and $z_{0} \geqslant z_{i}$. Then the solution curve through $X_{0}$ tends to a critical point on $C(\hat{z})$ as $\xi \rightarrow+\infty$ for some $\hat{z}>z_{0}$. 
Proof. Since $T^{\prime}(0)<0$, the solution moves into the region $T<T_{i}$ in positive time, and the $(w, z)$ equations decouple from the $(\tau, T)$ equations, i.e.

$$
\begin{cases}\mu \tau^{\prime}=F_{1}(\tau, T), & z^{\prime}=w, \\ \lambda T^{\prime}=\sigma \tau F_{2}\left(\tau, T_{1} z\right), & D w^{\prime}=-\sigma w .\end{cases}
$$

Assume for the moment that $T(\xi)<T_{i}$ for all $\xi>0$. Then $w(\xi)=w_{0} \exp \left(-\sigma^{-1} D \xi\right)$ so that

$$
z(\xi)=z_{0}+D \sigma^{-1} w_{0}\left[1-\exp \left({ }_{{ }^{\prime}} \sigma^{-1} D \xi\right)\right] .
$$

It follows that $z$ tends to the limit $\hat{z}=z_{0}+D \sigma^{-1} w_{0}$ and that $(\tau, T)$ converges to a $\operatorname{limit}(\hat{\tau}, \hat{T})$ on $F_{1}=0$ and $F_{2}(\tau, T, \hat{z})=0$.

Thus, it only remains to show that $T(\xi)<T_{i}$ for all $\xi>0$. Let $\xi_{1}>0$ be the first $\xi>0$ with $T(\xi)=T_{i}$. Since $z$ is monotone increasing on $\left[0, \xi_{1}\right], F_{2}(\tau, T, z)=0$ lies below $F_{2}\left(\tau, T, z_{0}\right)=0$. Since $T^{\prime}(0)<0$, it follows that $\tau_{0}>\tau_{1}$, where $\tau_{1}$ is as in Figure 16. Since $\tau^{\prime}>0$ along $\tau=\tau_{1}, T \leqslant T_{i}$, it follows that $\tau\left(\xi_{1}\right) \geqslant \tau_{1}$. Since $F_{2}\left(\tau, T_{i}, z\right)<0$ for $\tau \geqslant \tau_{1}$ and $z>z_{0}$, it follows that $T^{\prime}\left(\xi_{1}\right)<0$, which is a contradiction, thus $T(\xi)<T_{i}$ for all $\xi>0$.

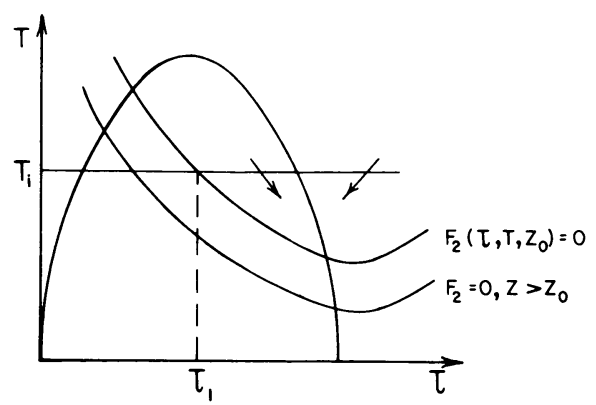

FigURE 16

Note that by Lemma 3.10 , the solution $\Re_{*}$ at $X_{0}$ satisfies the conditions of Lemma 6.1. Thus $\mathfrak{R}_{*}$ tends to a limit $C\left(z_{*}\right)$ at $+\infty$, where the limiting value $z_{*}$ of $z$ is $z_{*}=z_{0}+D \sigma^{-1} w_{0}$. Since $X_{0} \in N_{w}$, it follows that $z_{0} \leqslant \bar{z}$ and that $w_{0} \leqslant L$. Thus condition (9) of Theorem 2.1 implies that $z_{*}<1$, and $\mathfrak{N}_{*}$ is the solution curve $X_{w}(\xi)$.

Now let $\mathfrak{C}^{*}$ be the unstable manifold of $X_{L}^{*}$ with respect to the original equations, (6) $)_{0}$. This manifold is two-dimensional and it is easily seen by linearization that it contains solutions whose $z$ and $w$ components are positive near $X_{L}^{*}$. Moreover, the connecting orbit from $X_{L}^{*}$ to $X_{L^{*}}$ along which $z=w=0$ also lies in $\mathfrak{R}^{*}$. It follows that $\mathfrak{K}^{*}$ contains a solution $X_{0}(\xi)$ which closely approximates the connecting orbit and along which $z$ and $w$ are both positive. Since $\phi z>0$ for $T>T_{i}$ and $z>0$, it follows that $z$ must remain monotone increasing along $X_{0}(\xi)$ while $T>T_{i}$. If $X_{0}(\xi)$ is so close to the connecting orbit that it enters the excised neighborhood $B_{*}$ of $X_{L^{*}}$, it must eventually exit $A_{*}$, at which point it must be close 
to $\Re_{*}$. It follows that the orbit $X_{0}(\xi)$ hits a point $X_{0}$ which satisfies the hypotheses of Lemma 6.1. Thus $X_{0}(\xi)$ must tend to a limit $C\left(z_{0}\right)$ on $C(z)$ with $z_{0}$ near $z_{*}$, so that $z_{0}<1$. The projection on the $(T, z)$ plane is depicted in Figure 17.

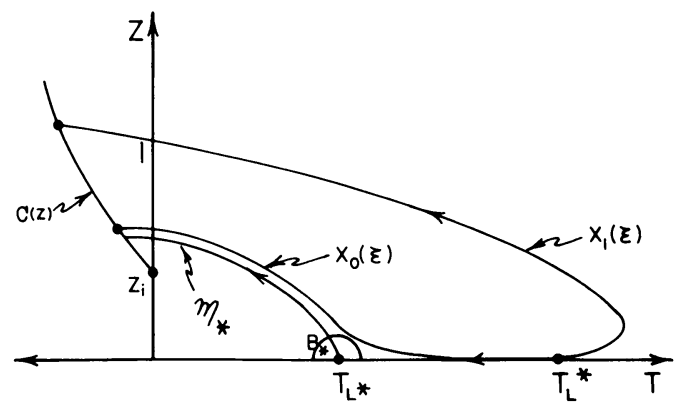

FIGURE 17

B. The orbit $X_{1}(\xi)$. We now invoke the results of $\S 5$ to construct a solution $X_{1}(\xi)$ in $\Re^{*}$ which tends to a critical point $C\left(z_{1}\right)$ on $C(z)$ with $z_{1}>1$.

LEMma 6.2. There exists a solution $X_{1}(\xi)$ in $\Re *$ which hits a point $\hat{X}$ at $\xi=0$ with $\hat{T}=T_{i}, \hat{z}=1, T^{\prime}(0)<0$, and $\hat{w} \geqslant 0$.

Proof. By the results of $\S 5$, there exists for all small $\delta>0$ a solution $X^{\delta}(\xi)$ in $S\left(N_{s}\right)$ of (6) which tends to $X_{L^{*}}$ as $\xi$ tends to $-\infty$. Thus $X^{\delta}(\xi)$ lies in $\Re^{*}$ when $T \geqslant T_{i}$. Moreover, $z$ and $w$ are positive along $X^{\delta}(\xi)$ while $T>T_{i}$. In order for $X^{\delta}(\xi)$ to remain in $N_{s}$, it follows that $T=T_{i}$ at some point along the orbit (otherwise, $z$ will increase without bound). At the first such point $X^{\delta}$, it follows from the construction of $N_{s}$ that $1-\delta<z^{\delta}<1, w^{\delta} \geqslant 0$, and $T^{\prime} \leqslant 0$. By (b) of Lemma 3.8, we also have that $T^{\prime}<0$ at this point.

Now $X^{\delta} \in N_{s}$ which is a compact set; thus $X^{\delta}$ (or some subsequence) tends to a limit, $X^{0}$. Since $\Re^{*}$ is closed, it follows that $X^{0} \in \Re^{*}$. From the above, it follows that $z^{0}=1, w^{0} \geqslant 0, T^{0}=T^{i}$, and that $T^{\prime} \leqslant 0$ at $X^{0}$. If $T^{\prime}=0$ at $X^{0}$, then by (b) of Lemma 3.8, $T$ has a local maximum at $T^{0}=T^{i}$, so that $T<T_{i}$ along the solution through $X^{0}$ in backward time. This contradicts the fact that $X^{\delta} \rightarrow X^{0}$ and that $T>T_{i}$ along $X^{\delta}(\xi)$ in backward time.

Thus the solution curve of $(6)_{0}$ through $X^{0}$ satisfies the requirements of Lemma 6.1 , and since $z^{0}=1$, the positive half orbit tends to a limit $C\left(z_{1}\right)$ on $C(z)$ for some $z_{1} \geqslant 1$.

Note that if $w^{0}=0$, then $X_{1}(\xi)$ is the desired connecting solution, $X_{s}(\xi)$, since $w=0, z=1$ is an invariant manifold of $(6)_{0}$ when $T \leqslant T_{i}$. However, this would imply that the 2-manifold $\mathfrak{K}^{*}$ intersects the 2-plane, $T=T_{i}, w=0$, in $\mathbf{R}^{4}$; generically, this will not be the case. Thus in the following, we assume that $w^{0}>0$, so that the limiting value $z_{1}$ of $z$ exceeds unity. 
C. Existence of $X_{s}(\xi)$. Suppose that $X_{0}(\xi)$ and $X_{1}(\xi)$ are parametrized so that $X_{0}(0)$ and $X_{1}(0)$ lie in $\partial A^{*}$, where $A^{*}$ is the neighborhood of $X_{L}^{*}$ used in the construction of $N_{s}$. Let $\Gamma(r)$ be a simple continuous curve in $\Re^{*}$ such that $\Gamma(r) \subset\{z>0, w>0\}, \Gamma(r) \subset A^{*}, \Gamma(0)=X_{0}(0)$, and $\Gamma(1)=X_{1}(0)$. Let $X_{r}(\xi)$ be the solution of $(6)_{0}$ which passes through $\Gamma(r)$ at $\xi=0$.

From previous remarks, it follows that either $X_{r}(\xi)$ tends to a limit $C\left(z_{r}\right)$ along $C(\xi)$ with $z_{r} \geqslant z_{i}$, or that $z$ becomes unbounded along $X_{r}$ in positive time. In particular, $z$ must be monotone increasing along $X_{r}$ while $T>T_{i}$. If $T>T_{i}$ along the entire orbit, then $X_{r}$ either tends to $C\left(z_{i}\right)$ or $z$ becomes unbounded in positive time. If $T=T_{i}$ at some point along $X_{r}$, then by arguments similar to those of Lemma 3.10, $X_{r}$ satisfies the conditions of Lemma 6.1 when $T=T_{i}$, and the solution tends to a critical point $C\left(z_{r}\right)$ with $z_{r}>z_{i}$. Note that in either case, $z$ is monotone increasing along the entire orbit.

The proof of Theorem 2.1 is completed as follows. Let

$A=\left\{r \in[0,1]: X_{r}(\xi)\right.$ tends to a critical point $C\left(z_{r}\right)$ with $\left.z_{r}<1\right\}$,

$B=\left\{r \in[0,1]\right.$ : the $z$-component $z_{r}(\xi)$ equals 1 at some point along $\left.X_{r}(\xi)\right\}$.

Assume that the connecting orbit $X_{s}(\xi)$ from $X_{L}^{*}$ to $X_{R}=C(1)$ does not exist. We claim that the following hold:

(i) $A \cup B=[0,1]$;

(ii) $A$ and $B$ are both open;

(iii) $A$ and $B$ are nonempty.

The last assertion is clear, since $0 \in A$ and $1 \in B$.

To prove (i), assume that $r \notin B$, so that $z \leqslant 1$ along the entire orbit, $X_{r}$. From previous remarks, it follows that $X_{r}$ tends to a limit $C\left(z_{r}\right)$ with $z_{r} \leqslant 1$, and by hypothesis, $z_{r}<1$. Thus $r \in A$.

Next, suppose that $r_{0} \in A$. If $T \geqslant T_{i}$ along the entire orbit, then $X_{r_{0}}$ tends to $X_{i}$. Thus $X_{r_{0}}$ eventually enters the neighborhood $A_{i}$ of $X_{i}$ in positive time. It follows for $r$ sufficiently near $r_{0}$ that $X_{r}$ also enters $A_{i}$. If $T \geqslant T_{i}$ along the entire orbit $X_{r}$, then $X_{r}$ also tends to $X_{i}$, since $A_{i}$ is an attracting neighborhood relative to the region $\left\{T \geqslant T_{i}\right\}$. If this is not the case, then $T=T_{i}$ at some point along $X_{r}$ while $X_{r} \in A_{i}$. By condition (9), it follows that $X_{r}$ tends to a critical point $z_{r}$ with $z_{r}<1$. Finally, suppose that $T=T_{i}$ at some point along $X_{r_{0}}$, say, at $\xi=\xi_{0}>0$. If $r$ is sufficiently near $r_{0}$, it follows that $X_{r}$ hits a point with $T=T_{i}$ near the time $\xi=\xi_{0}$, at which point $X_{r}$ will be close to $X_{r_{0}}$. It again follows from (9) that $X_{r}$ tends to a critical point $C\left(z_{r}\right)$ with $z_{r}$ near $z_{r_{0}}$, and hence, less than unity. Hence $A$ is open.

Finally, we show that $B$ is open. Let $r_{0} \in B$, and suppose that $z=1$ at some point with $T>T_{i}$ along $X_{r_{0}}$. It follows that $z^{\prime}>0$ at this point (since $\phi>0$ in this region). It again follows from standard continuous dependence theorems that $X_{r}$ hits a point with $z=1$ and $T>T_{i}$, and that at this point $z^{\prime}>0$. Thus $r \in B$ for $r$ sufficiently near $r_{0}$.

If $z=1$ when $T \leqslant T_{i}$ along $X_{r_{0}}$, then either $z^{\prime}>0$ (in which case the preceding argument applies), or $z^{\prime}=w=0$. In the latter case, the solution would be a connection from $X_{L}^{*}$ to $X_{R}$ (since $z \equiv 1$ in forward time), contrary to our hypothesis.

Since (i), (ii), and (iii) contradict the connectedness of $[0,1]$, we see that the solution $X_{s}(\xi)$ does in fact exist. 
Finally, note that these results hold for all $\eta>0$ (cf. Figure 1). It easily follows that the solutions $X_{s}(\xi)$ for $\eta>0$ tend to a similar connecting orbit as $\eta$ approaches zero.

D. Chapman-Jouget detonation. Our results apply to the case where $\left(\sigma, q_{0}\right)$ lies in the interior of the shaded region in Figure 0 . It follows that the sound speed of the reaction products exceeds the wave velocity, $\sigma$. This will be the case when the detonation is initiated by a violent disturbance, e.g., an explosion. Another situation where this will occur is when a combustion wave proceeds from a pipe of large diameter to a pipe of small diameter.

Detonations can also arise spontaneously. For example, suppose that the gas is ignited sufficiently so that a combustion wave is formed. Initially, the wave speed may be subsonic relative to the unburned gas. However, if energy losses due to dissipative effects are not too great, the wave velocity will be accelerated; the asymptotic velocity is then determined by the curve $q_{0}=\bar{q}_{0}(\sigma)$ in Figure 0 . This is called Chapman-Jouget detonation. For a given $q_{0}$, let $\sigma_{C J}$ be the corresponding wave velocity on this curve.

It is easily seen that Theorem 2.1 is valid when $\sigma=\sigma_{C J}$. First note that as $\sigma \downarrow \sigma_{C J}$, the critical points $X_{L^{*}}$ and $X_{L}^{*}$ coalesce to a single critical point, $X_{C J}$. Moreover, as $\sigma$ decreases, it is easily checked that $T_{L^{*}}$ increases. Thus the temperature $T_{C J}$ at $X_{C J}$ exceeds $T_{L^{*}}$, and hence, $T_{i}$. (This can be seen by noting that when $\sigma=\sigma_{C J}, F_{1}=0$ is tangent to $F_{2}(\tau, T, 0)=0$.) Let $z_{i}(\sigma)$ be the value of $z$ at $X_{i}$ for a given $\sigma$. Then $z_{i}(\sigma)$ increases to a limiting value $z_{i}\left(\sigma_{C J}\right)<1$ as $\sigma \downarrow \sigma_{C J}$.

Suppose that $\bar{z} \in\left(z_{i}\left(\sigma_{C J}\right), 1\right)$, where $\bar{z}$ is as in $\S 3$.A. It is then easily seen that the conditions (7), (8) and (9) are not affected as $\sigma \downarrow \sigma_{C J}$. In particular, these conditions are used in estimates of solutions in regions of phase space where $X$ is far away from $X_{L^{*}}$ and $X_{L}^{*}$ (see Figures 4, 7, and 13). It follows that for each $\sigma<\sigma_{C J}$, a strong detonation $X_{s}^{\sigma}(\xi)$ from $X_{L}^{*}$ to $X_{R}$ exists, where the conditions (7), (8), and (9) hold uniformly, as $\sigma \downarrow \sigma_{C J}$. It easily follows the solutions $X_{s}^{\sigma}(\xi)$ tend to an orbit $X_{C J}(\xi)$ which connects $X_{C J}$ to $X_{R}$.

E. Concluding remarks. It has been assumed that $\left(\sigma, q_{0}\right)$ are such that $T_{i}<T_{L^{*}}<$ $T_{L}^{*}$. As mentioned in the introduction, other possible cases are that $T_{L^{*}}<T_{i}<T_{L}^{*}$ or $T_{L^{*}}<T_{L}^{*}<T_{i}$. In the former case, weak detonations do not exist. The construction of $N_{s}$ and of the orbit $X_{1}(\xi)$ is considerably simpler, and with minor modifications, the methods used here will be applicable. The latter case, however, is quite different. Since $\phi=0$ for $T \leqslant T_{i}$, connecting orbits from $X_{L}^{*}$ to $X_{R}$ do not exist. However, there now exists a curve of rest points $C(T)$ of $(6)_{0}$ with $T_{L}^{*}<T \leqslant T_{i}$ and whose $z$-components are positive. Thus the gas may only be partially burned at $\xi=-\infty$. This phenomenon was first noticed by Majda and Rosales [7], where asymptotic solutions were produced with this type of behavior. It is plausible that the method used here should be applicable to this case (i.e., an index argument for a modified flow followed by a shooting argument); however, the constructions would be different, since the "correct" critical point along $C(T)$ to which the orbit should tend at $-\infty$ is not a priori known.

Finally, we remark that for strong shocks, $\lambda$ and $\mu$ may depend on the fluid variables. However, if $\lambda_{0}$ and $\mu_{0}$ are positive constants which majorize $\lambda$ and $\mu$ and 
which satisfy (7) and (8), the $\tau^{\prime}$ and $T^{\prime}$ equations can be written in the form

$$
\lambda_{0} T^{\prime}=\lambda_{0} \lambda^{-1} \sigma \tau F_{2}, \quad \mu_{0} \tau^{\prime}=\mu_{0} \mu^{-1} F_{1}
$$

since the coefficients $\lambda_{0} \lambda^{-1}$ and $\mu_{0} \mu^{-1}$ exceed unity, the conditions (7)-(9) of Theorem 2.1 hold for these new nonlinearities. The proof is then the same as before. This can be seen by noting that higher derivatives of $\tau$ or $T$ need to be computed only when $F_{1}$ or $F_{2}$ equals zero. Thus the (nonzero) derivatives of these new coefficients will be multiplied $F_{1}$, or $F_{2}$, and so they will not actually be present in such computations.

Appendix 1. Derivation of (6). The equations (6) are obtained from (5) as follows. From (5a) and (2), (5b) can be written as

$$
-\mu \sigma \tau^{\prime}=\tau\left[\sigma^{2}\left(\tau-\tau_{R}\right)+p-p_{R}\right]=\sigma^{2} \tau^{2}-A \tau+R T,
$$

where $A=\sigma^{2} \tau_{R}^{2}+p_{R}$. Similarly, (5c) can be rewritten (using the above, (5a), (2), and (3)) as

$$
\begin{aligned}
\lambda T^{\prime}=\mu \sigma u \tau^{\prime}+\tau\left\{-\sigma\left[c T-c T_{R}+q_{0}(z-1)\right]\right. & \left.\quad+\sigma^{2}\left(\tau-\tau_{R}\right)\left(u+u_{R}\right) / 2+p u-p_{R} u_{R}\right\} \\
=\tau\left\{-u\left[\sigma^{2}\left(\tau-\tau_{R}\right)+p-p_{R}\right]-\right. & \sigma\left[c T-c T_{R}+q_{0}(z-1)\right] \\
& \left.+\sigma^{2}\left(\tau-\tau_{R}\right)\left(u+u_{R}\right) / 2+p u-p_{R} u_{R}\right\} \\
= & \tau\left\{\left(\tau-\tau_{R}\right)\left[-\sigma^{2}\left(u-u_{R}\right) / 2-\sigma p_{R}\right]-\sigma c T+\sigma c T_{R}-\sigma q_{0}(z-1)\right\} \\
=\sigma \tau\left[\frac{\sigma^{2}}{2}\left(\tau-\tau_{R}\right)^{2}-p_{R}\left(\tau-\tau_{R}\right)+c T_{R}-c T-q_{0}(z-1)\right] &
\end{aligned}
$$

where $A$ is as above and $B=\sigma^{2} \tau^{2} R / 2+p_{R} \tau_{R}+c T_{R}$.

Appendix 2. The critical points of (6). Let $\bar{X} \neq X_{R}$ be a critical point of (6). Then $\bar{w}=0, \Phi(\bar{T}, \bar{z}) \bar{z}=0$, and $F_{1}=F_{2}=0$ (the critical points with $\tau=T=0$ will not enter into the discussion).

Lemma A.1. There are exactly four critical points of (6), $X_{R}, X_{L^{*}}, X_{L}^{*}$, and $X_{i}$, provided that $\left(\sigma, q_{0}\right)$ lies in the shaded region in Figure 0.

Proof. If $\bar{X}$ is a critical point, it follows that

$$
\begin{aligned}
-\sigma\left(\bar{\tau}-\bar{\tau}_{R}\right) & =\bar{u}-u_{R}, \\
-\sigma\left(\bar{u}-u_{R}\right) & =-\left(\bar{p}-p_{R}\right), \\
-\sigma\left(\bar{e}-e_{R}\right)+\left(\bar{u}^{2}-u_{R}^{2}\right) / 2 & =-\left(\bar{p} \bar{u}-p_{R} u_{R}\right),
\end{aligned}
$$

where $\bar{p}$ and $\bar{e}$ are determined from (2) and (3), and $\bar{u}$ is determined by $\sigma, \tau_{R}, \bar{\tau}$, and $u_{R}$. It follows that if $H$ is the Hugoniot function,

$$
H(\tau, p)=\bar{e}-e_{R}+\left(p+p_{R}\right)\left(\tau-\tau_{R}\right) / 2,
$$


then $H(\bar{\tau}, \bar{p})=q_{0}(1-\bar{z})$ (cf. Courant and Friedrichs [4, p. 208]). Moreover, $(\bar{\tau}, \bar{p})$ must also satisfy $\bar{p}-p_{R}=-\sigma^{2}\left(\bar{\tau}-\tau_{R}\right)$. It follows that $(\bar{\tau}, \bar{p})$ must lie on the intersection of this chord with the level curves of $H$.

It is easily checked that the hyperbola $\tau p=R T_{i}$ has the aspect indicated in Figure A.1. Thus for sufficiently large $\sigma$, there is determined a range of admissible $q_{0}$, namely $q_{0}(\sigma) \leqslant q_{0} \leqslant \bar{q}_{0}(\sigma)$ for critical points with $\bar{z}=0$. (This is due to the hypothesis that the temperature at such a critical point must exceed $T_{i}$.) Thus there are precisely two such critical points, which we denote by $X_{L^{*}}$ and $X_{L}^{*}$.

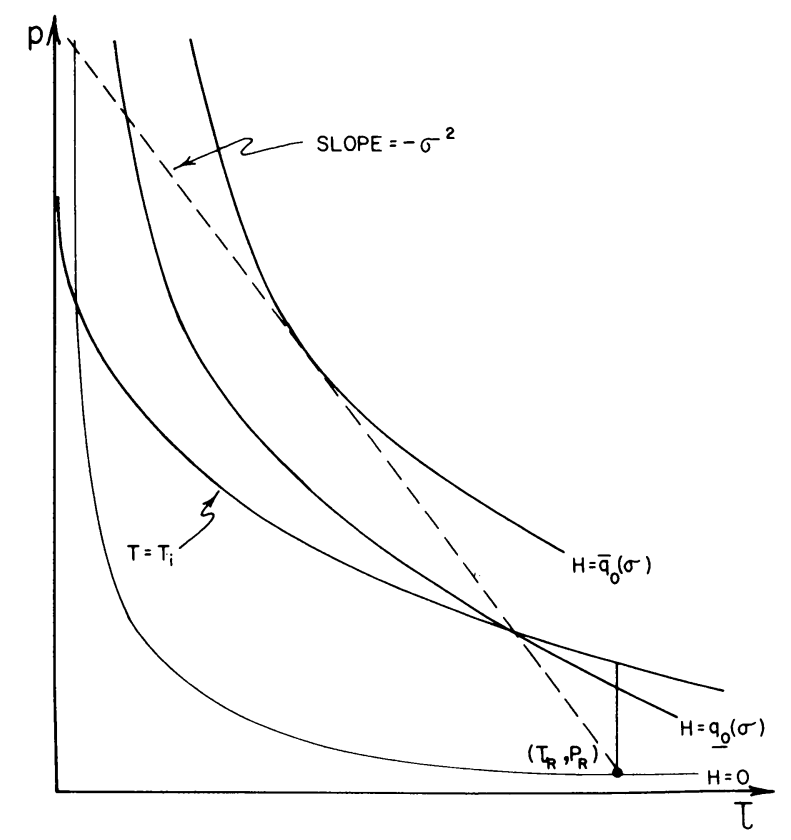

Figure A.1

If $\bar{z}>0$, then either $\bar{T}=T_{i}$ or $\bar{z}=1$ and $\bar{T} \leqslant T_{i}$ (see Figure 1). Let $z_{i}$ be defined by $q_{0}(\sigma)=q_{0}\left(1-z_{i}\right)$. It follows that there is precisely one critical point when $T=\bar{T}_{i}$ and $z=z_{i}$ (which we denote by $X_{i}$ ), and one critical point with $z=1$ and $T \leqslant T_{i}$ (namely $X_{R}$ ).

It is easily checked that $\bar{q}_{0}(\sigma)$ tends to $+\infty$ as $\sigma \rightarrow+\infty$, while $\underline{q_{0}}(\sigma)$ tends to a finite limit.

We next show that $T_{i}<T_{L^{*}}<T_{L}^{*}$, and that the curves $F_{2}=0$ have the aspect indicated in Figure 2. These facts follow from the next lemma.

Lemma A.2. Suppose that $\left(\sigma, q_{0}\right)$ lies in the shaded region in Figure 0 , and that $(\hat{\tau}, \hat{T})$ is the point on $F_{2}(\tau, T, 1)=0$ where the temperature is minimal. Then $\hat{T}>0$, and $(\hat{\tau}, \hat{T})$ lies to the right of the right branch of $F_{1}=0$.

Proof. The curve $F_{2}(\tau, T, 1)=0$ is

$$
c T=\frac{\sigma^{2}}{2} \tau^{2}-A \tau+B=\frac{\sigma^{2}}{2}\left(\tau-\tau_{R}\right)^{2}-p_{R}\left(\tau-\tau_{R}\right)+c T_{R}
$$


so that $\hat{T}$ is minimal when $\hat{\tau}=A / \sigma^{2}$. Thus $\hat{\tau}$ is the positive root of $F_{1}=0$. The lemma will be proved if it can be shown that $\hat{T}>0$.

If $\tau$ is such that $F_{2}(\tau, 0,1)=0$, then

$$
\tau-\tau_{R}=\frac{p_{R} \pm \sqrt{p_{R}^{2}-2 \sigma^{2} c T_{R}}}{\sigma^{2}} ;
$$

$\tau$ will be complex if the discriminant is negative. If $p=p(\tau)$ is the Hugoniot curve $H=0$ in Figure A.1, then $p^{\prime}\left(\tau_{R}\right)>-\sigma^{2}$. Moreover, from (2) and (3) it follows that $p^{\prime}\left(\tau_{R}\right)=-\left(R C^{-1}+1\right) p_{R} / \tau_{R}$, and that

$$
\begin{aligned}
P_{R}^{2}-2 \sigma^{2} c T_{R} & <p_{R}^{2}-2 p^{\prime}\left(\tau_{R}\right) c T_{R}<p_{R}^{2}-2(R+c) p_{R} T_{R} / \tau_{R} \\
& =p_{R}^{2}-2(R+c) p_{R}^{2} / R<0 .
\end{aligned}
$$

Thus $F_{2}(\tau, T, 1)=0$ has no real roots. This completes the proof.

Appendix 3. The indices of the critical points. The index of a hyperbolic critical point is a $k$-sphere, $\Sigma^{k}$, when $k$ is the dimension of its unstable manifold. Thus $k$ can be determined by linearization.

Lemma A.3. The index of $X_{R}$ is $\Sigma^{1}$.

Proof. Let

$$
\begin{array}{ll}
a=\mu^{-1} \frac{\partial F_{1}}{\partial \tau}, & b=\mu^{-1} \frac{\partial F_{1}}{\partial T}, \\
c=\lambda^{-1} \sigma \tau \frac{\partial F_{2}}{\partial \tau}, & d=\lambda^{-1} \sigma \tau \frac{\partial F_{2}}{\partial T},
\end{array}
$$

where the partials are evaluated at $\left(\tau_{R}, T_{R}, 1\right)$. It is easily checked that $a, b, c$, and $d$ are all negative (see Figure 2(a)).

The linearization of (6) about $X_{R}$ is therefore

$$
M=\left[\begin{array}{cccc}
a & b & 0 & 0 \\
c & d & -L & 0 \\
0 & 0 & 0 & 1 \\
0 & 0 & \rho & -\Gamma
\end{array}\right],
$$

where $L=\lambda^{-1} \sigma \tau_{R} q_{0}<\rho=\varepsilon^{-1} \Phi_{z}\left(T_{R}, 1\right)>0$ and $\Gamma=\sigma \varepsilon^{-1}>0$.

It is easily verified that the characteristic polynomial is

$$
c(s)=\operatorname{det}(M-s I)=\left(s^{2}-(a+d) s+a d-b c\right)\left(s^{2}+\Gamma s-\rho\right) .
$$

Since $a, b, c$, and $d$ are negative, the first factor has roots with negative real part. The second factor has roots $-\Gamma \pm \sqrt{\Gamma^{2}+4 \rho}$; since $\rho$ and $\Gamma$ are positive, this term contributes one positive and one negative root.

Lemma A.4. The index of $X_{L^{*}}$ is $\Sigma^{1}$ and the index of $X_{L}^{*}$ is $\Sigma^{2}$.

Proof. In both cases the linearization has the form (A.1), where $\rho=D^{-1} \phi$ and $\Gamma=D^{-1} \sigma$. Thus $\rho$ and $\Gamma$ are still positive.

In the case of $X_{L^{*}}, a, b, c$, and $d$ are all negative, so the argument is the same as for $X_{R}$. 
At $X_{L}^{*}$, it will be shown that the first factor in $c(s)$ above has one positive and one negative root. To this end, note that the slope $-a / b$ of the tangent to $F_{1}=0$ is greater than the slope $-c / d$ to the tangent to $F_{2}=0$ at $X_{L^{*}}$ (see Figure 2(c)). Since $b$ and $d$ are both negative, it follows that $a d-b c<0$. The first factor in $c(s)$ therefore contributes one positive and one negative root. This completes the proof.

LEMmA A.5. The index of $X_{i}$ is $\Sigma^{0}$, i.e., $X_{i}$ is an attractor.

Proof. The linearization of (6) about $X_{i}$ is

$$
M=\left[\begin{array}{cccc}
a & b & 0 & 0 \\
c & d & -L & 0 \\
0 & 0 & 0 & 1 \\
0 & A & 0 & -\Gamma
\end{array}\right]
$$

here $L=\lambda^{-1} \sigma \tau_{i} q_{i}, A=D^{-1} \Phi_{T}\left(T_{i}, z_{i}\right) z_{i}>0$, and $\Gamma=D^{-1} \sigma$. Also, $a, b, c$ and $d$ are all negative.

It is easily shown that

$$
c_{A}(s)=\operatorname{det}(M-s I)=\left(s^{2}-(a+d) s+a d-b c\right)\left(s^{2}+s \Gamma\right)-(a-s) A L .
$$

Suppose first that $A=0$. Since $a, b, c$, and $d$ are all negative and $\Gamma$ is positive, it follows that $c(s)$ has three roots with negative real part, and one zero root. Moreover, $\partial c_{A}(0) / \partial A>0$, so that $c_{A}(0)>0$ for small positive $A$. It follows that for such $A, c_{A}(s)$ has four roots with negative real part.

Now let $A$ assume larger values. If for some $A>0, c_{A}(s)=0$ for some $s=i q$ with $q \in R$, it follows that

$$
\operatorname{Im} c_{A}(i q)=-(a+d) q^{3}+[(a d-b c)+A L] q=0,
$$

or $q^{2}=[a d-b c+A L](a+d)^{-1}$. Since $a d-b c>0, A L>0$, and $a+d<0$, it follows that this equation has no real solutions. Thus $c_{A}(s)$ has roots with negative real part for all $A>0$.

This completes the proof.

Appendix 4. The Conley index. Given a flow on $\mathbf{R}^{n}$, let $N$ be a compact neighborhood in $\mathbf{R}^{n}$ and let $S(N)$ be the maximal invariant set in $N$. ( $S(N)$ consists of all orbits which stay in $N$ for all time.) If $S(N)$ is interior to $N$ then $N$ is called an isolating neighborhood and $S(N)$ is called an isolated invariant set.

A homotopy invariant associated with $S(N)$ is defined as follows. Let $N_{1}$ and $N_{2}$ be subsets of $N$ with the following properties:

(i) $N_{i}$ is positively invariant relative to $N, i=1,2$.

(ii) $N_{1} \backslash N_{2}$ contains a neighborhood of $S(N)$.

(iii) Solution curves which leave $N$ in positive time hit points in $N_{2}$ before leaving $N$.

Then $\left(N_{1}, N_{2}\right)$ is called an index pair. If $S(N)$ is an isolated invariant set then index pairs exist (see Conley [1]). Without loss of generality, it can be assumed that $N_{2} \subset N_{1}$. 
The Conley index of $S(N)$, denoted by $h(S(N))$, is defined to be the homotopy type of $N_{1} / N_{2}$; i.e., the space obtained by collapsing $N_{2}$ to a point. Thus $h(S(N))=$ $\left[N_{1} / N_{2}\right]$. This homotopy type is the same for all index pairs and is invariant under deformations of the flow, provided that $S(N)$ is interior to $N$ throughout the homotopy (see Conley [1]).

The simplest case is when $S(N)=\varnothing$. An index pair is determined by $N_{1}=N_{2}=N$ so that $h(S(N))$ is $\overline{0}=[N / N]$, i.e., the homotopy type of a pointed point.

Next, consider the one-dimensional flow generated by $\dot{x}=x$. If $N=[-1,1]$, then $N$ is an isolating neighborhood for $S(N)=\{0\}$. Also, $N_{1}=N, N_{2}=\{-1,1\}$ is an index pair, so that $h(S(N))=\Sigma^{1}$, i.e., a pointed one-sphere.

In general, the index of a hyperbolic critical point is $\Sigma^{k}$, where $k$ is the number of positive eigenvalues of the linearized equations.

If $S_{1}$ and $S_{2}$ are disjoint isolated invariant sets, then $h\left(S_{1} \cup S_{2}\right)=h\left(S_{1}\right) \vee h\left(S_{2}\right)$. This is the space obtained by glueing $h\left(S_{1}\right)$ and $h\left(S_{2}\right)$ together at their distinguished point; see Conley [1].

Suppose that $N$ is an isolating neighborhood which contains exactly two hyperbolic critical points. If $h(S(N))=\overline{0}$, then $S(N)$ must contain nonconstant solutions, since $\overline{0}$ is a different homotopy type than $\Sigma^{k} \vee \Sigma^{l}$ for all $k, l \geqslant 0$. Moreover, it follows from the sum formula that neither critical point is isolated in $S(N)$.

\section{REFERENCES}

1. C. Conley, Isolated invariant sets and the generalized Morse index, C.B.M.S. Regional Conf. Series in Math., No. 38, Amer. Math. Soc., Providence, R.I., 1978.

2. C. Conley and J. Smoller, Shock waves as limits of progressive wave solutions of higher order equations, Comm. Pure Appl. Math. 24 (1971), 459-472.

3. _ On the structure of magnetohydrodynamic shock waves, Comm. Pure Appl. Math. 27 (1974), $367-375$.

4. R. Courant and K. O. Friedrichs, Supersonic flow and shock waves, Springer, New York, 1948.

5. P. Fife, Propagating fronts in reactive media, preprint.

6. A. Majda, A qualitative model for dynamic combustion, SIAM J. Appl. Math. 41 (1981), 70-93.

7. A. Majda and R. Rosales, Weakly nonlinear detonation waves, preprint.

8. J. A. Smoller and R. Shapiro, Dispersion and shock wave structure, preprint.

9. F. Williams, Combustion theory, Addison-Wesley, Reading, Mass., 1965.

10. Ia. B. Zeldovich and A. S. Kampaneets, The theory of detonation, Academic Press, New York, 1960.

Department of Mathematics, University of Massachusetts, Amherst, Massachusetts 01003 